\title{
A BOUND ON THE GEOMETRIC GENUS OF PROJECTIVE VARIETIES VERIFYING CERTAIN FLAG CONDITIONS
}

\author{
VINCENZO DI GENNARO
}

\begin{abstract}
Fix integers $n, r, s_{1}, \ldots, s_{l}$ and let $\mathcal{S}\left(n, r ; s_{1}, \ldots, s_{l}\right)$ be the set of all integral, projective and nondegenerate varieties $V$ of degree $s_{1}$ and dimension $n$ in the projective space $\mathbf{P}^{r}$, such that, for all $i=2, \ldots, l, V$ does not lie on any variety of dimension $n+i-1$ and degree $<s_{i}$. We say that a variety $V$ satisfies a flag condition of type $\left(n, r ; s_{1}, \ldots, s_{l}\right)$ if $V$ belongs to $\mathcal{S}\left(n, r ; s_{1}, \ldots, s_{l}\right)$. In this paper, under the hypotheses $s_{1}>>\ldots>>s_{l}$, we determine an upper bound $G^{h}\left(n, r ; s_{1}, \ldots, s_{l}\right)$, depending only on $n, r, s_{1}, \ldots, s_{l}$, for the number $G\left(n, r ; s_{1}, \ldots, s_{l}\right):=\max \left\{p_{g}(V): V \in \mathcal{S}\left(n, r ; s_{1}, \ldots, s_{l}\right)\right\}$, where $p_{g}(V)$ denotes the geometric genus of $V$. In case $n=1$ and $l=2$, the study of an upper bound for the geometric genus has a quite long history and, for $n \geq 1, l=2$ and $s_{2}=r-n$, it has been introduced by Harris. We exhibit sharp results for particular ranges of our numerical data $n, r, s_{1}, \ldots, s_{l}$. For instance, we extend Halphen's theorem for space curves to the case of codimension two and characterize the smooth complete intersections of dimension $n$ in $\mathbf{P}^{n+3}$ as the smooth varieties of maximal geometric genus with respect to appropriate flag condition. This result applies to smooth surfaces in $\mathbf{P}^{5}$. Next we discuss how far $G^{h}\left(n, r ; s_{1}, \ldots, s_{l}\right)$ is from $G\left(n, r ; s_{1}, \ldots, s_{l}\right)$ and show a sort of lifting theorem which states that, at least in certain cases, the varieties $V \in \mathcal{S}\left(n, r ; s_{1}, \ldots, s_{l}\right)$ of maximal geometric genus $G\left(n, r ; s_{1}, \ldots, s_{l}\right)$ must in fact lie on a flag such as $V=V_{s_{1}}^{n} \subset V_{s_{2}}^{n+1} \subset \ldots \subset V_{s_{l}}^{n+l-1} \subset \mathbf{P}^{r}$, where $V_{s}^{j}$ denotes a subvariety of $\mathbf{P}^{r}$ of degree $s$ and dimension $j$. We also discuss further generalizations of flag conditions, and finally we deduce some bounds for Castelnuovo's regularity of varieties verifying flag conditions.
\end{abstract}

\section{INTRODUCTION}

Fix integers $n, r, s_{1}, \ldots, s_{l}$ such that $1 \leq n \leq r-2,2 \leq l \leq r-n$, and let $\mathcal{S}\left(n, r ; s_{1}, \ldots, s_{l}\right)$ be the set of all integral, projective and nondegenerate varieties $V$ of degree $s_{1}$ and dimension $n$ in the projective space $\mathbf{P}^{r}$, such that, for all $i=2, \ldots, l$, $V$ does not lie on any variety of dimension $n+i-1$ and degree $<s_{i}$. Following [CCD2], we say that a variety $V$ satisfies a flag condition of type $\left(n, r ; s_{1}, \ldots, s_{l}\right)$ if $V$ belongs to $\mathcal{S}\left(n, r ; s_{1}, \ldots, s_{l}\right)$. In this paper, under the hypotheses $s_{1}>>\ldots>>s_{l}$, we determine an upper bound $G^{h}\left(n, r ; s_{1}, \ldots, s_{l}\right)$, depending only on $n, r, s_{1}, \ldots, s_{l}$, for the number

$$
G\left(n, r ; s_{1}, \ldots, s_{l}\right):=\max \left\{p_{g}(V): V \in \mathcal{S}\left(n, r ; s_{1}, \ldots, s_{l}\right)\right\},
$$

Received by the editors October 23, 1995.

1991 Mathematics Subject Classification. Primary 14J99, 14M07, 14M10; Secondary 14F17.

Key words and phrases. Projective varieties, geometric genus, arithmetic genus, flag conditions, Castelnuovo's theory, low codimension varieties, complete intersections, Castelnuovo's regularity. 
where $p_{g}(V)$ denotes the geometric genus of (a desingularization of) $V$ (see Theorem (2.5) and, for the special case $l=r-n$, Theorem (4.1)). Note that $G\left(n, r ; s_{1}, \ldots, s_{l}\right)$ is a finite number by a general result of [Hr2].

In the case of curves, i.e. when $n=1$, we refer to [CCD2] for an overview on this question and related topics. Here we only notice that, when $n=1$ and $l=2$, the study of an upper bound for the geometric genus has a quite long history (see [Ha], [No], [Ca], [GH], [ACGH], [Ht], [GP], [Hr1], [EH], [Ci1], [CCD1], [CCD2]) and that, for $n \geq 1, l=2$ and $s_{2}=r-n$, it has been introduced by Harris [Hr2]. As well as in $[\mathrm{D}]$, where we generalize to the case $n \geq 1$ some of the results obtained in [CCD1] for $n=1$ and $l=2$, in the present article we partly extend [CCD2] and [CCD3] to the case $n \geq 1$.

Let us now discuss our results in more detail.

We begin in Section 1 by establishing our notation and conventions. Here we define the functions $G^{h}\left(n, r ; s_{1}, \ldots, s_{l}\right)$ and make explicit what the condition $s_{1}>>$ $\ldots>>s_{l}$ means. Our main result, i.e. Theorem (2.5), appears in Section 2 and claims that $G^{h}\left(n, r ; s_{1}, \ldots, s_{l}\right)$ is in fact an upper bound for $G\left(n, r ; s_{1}, \ldots, s_{l}\right)$. In order to show this theorem, we need some numerical properties of the functions $G^{h}\left(n, r ; s_{1}, \ldots, s_{l}\right)$ which are essential in the rest of the paper and whose proof we exhibit in the Appendix. The first, i.e. Proposition A, is a result on the asymptotic behaviour of the functions $G^{h}\left(n, r ; s_{1}, \ldots, s_{l}\right)$, which enables us to obtain certain properties of monotonicity of these functions, i.e. Proposition B. It is worth remarking the delicate role which the properties of monotonicity of the functions $G\left(1,3 ; s_{1}, s_{2}\right)$ historically play in the study of space curves [Ha], [GP]. For instance, we recall Halphen's principle, which claims that $G\left(1,3 ; s_{1}, s_{2}+1\right)<G\left(1,3 ; s_{1}, s_{2}\right)$ [E]. In Corollary (4.9) we extend such a principle to the functions $G\left(n, r ; s_{1}, \ldots, s_{l}\right)$ we introduced in (0.1), at least for particular ranges of $n, r, s_{1}, \ldots, s_{l}$.

Next, Sections 3 and 4 are devoted to analysing the sharpness of the bound $G^{h}\left(n, r ; s_{1}, \ldots, s_{l}\right)$. First in Section 3 we specialize our study to the case of codimension two, i.e. $r-n=2$ (and, a fortiori, $l=2$ ). Here we extend to codimension two Halphen's theorem for space curves (see [Ha], [GP] and our Theorem (3.1)). This gives an answer to a question in [Ci3, p.37], improves [NV, Theorem 4] and [N, Theorem (5.4)] for $s_{1}>s_{2}^{2}-s_{2}$, and also improves the analysis of the case $l=2$ introduced in [D]. In particular it follows that, for $s_{1}>s_{2}^{2}-s_{2}$, the bound $G^{h}\left(n, n+2 ; s_{1}, s_{2}\right)$ is sharp, i.e. $G^{h}\left(n, n+2 ; s_{1}, s_{2}\right)=G\left(n, n+2 ; s_{1}, s_{2}\right)$ (see also [D] for other ranges of $n, r, s_{1}$ and $s_{2}$ for which $G^{h}\left(n, r ; s_{1}, s_{2}\right)$ is sharp). We also point out that, when $n \geq 4$, any variety $V \in \mathcal{S}\left(n, n+2 ; s_{1}, s_{2}\right)$ of maximal geometric genus, not a complete intersection, must be singular (at least when $s_{1}>>s_{2}$, and $s_{2}=2$ or the remainder of the division of $s_{1}-1$ by $s_{2}$ is different from $0,1, s_{2}-3$ (Theorem (3.1), (iii))).

On the other hand, it is known that, when $l \geq 3, G^{h}\left(1, r ; s_{1}, \ldots, s_{l}\right)$ is necessarily not sharp (see [CCD2, Remark (2.5)] and (1.5)). This leads us to expect that also $G^{h}\left(n, r ; s_{1}, \ldots, s_{l}\right)$ is not sharp, for $n \geq 2$ and $l \geq 3$. However, by using the results for curves in $\mathbf{P}^{4}$ proved in [CCD3], in Section 4 we refine our upper bound in the special case $l=r-n \geq 3$. Hence we define a more subtle upper bound $G^{\tilde{h}}\left(n, r ; s_{1}, \ldots, s_{r-n}\right)$, which we prove to be sharp when $l=r-n=3, s_{1}>>s_{2}>>$ $s_{3}$ and $s_{2}$ divides $s_{1}$ (Theorem (4.2)). Next we obtain a characterization of the smooth complete intersections of type $(a, b, c)$ in $\mathbf{P}^{n+3}$, with $a>>b>>c$, as the smooth varieties of maximal geometric genus with respect to the appropriate flag 
conditions $(n, n+3 ; a b c, b c, c)$ (Theorem (4.3)). Notice that this result applies to smooth surfaces in $\mathbf{P}^{5}$ (in this case, see Theorem (4.4) for a more general statement), and that the analogous property in codimension two is a particular case of our extension of Halphen's theorem (i.e. Theorem (3.1)). Also note that, in the ranges where we exhibit sharp results, all the varieties $V$ verifying flag conditions and of maximal geometric genus can have only weak singularities, in the sense that they verify the equality $p_{g}(V)=h^{0}\left(V, \omega_{V}\right)$, where $\omega_{V}$ denotes the dualizing sheaf of $V$ (in general, one has $p_{g}(V) \leq h^{0}\left(V, \omega_{V}\right)$ (Lemma (2.2))).

At the end of Section 4 we address the problem of how far $G^{h}\left(n, r ; s_{1}, \ldots, s_{l}\right)$ is from $G\left(n, r ; s_{1}, \ldots, s_{l}\right)$ and give a numerical estimate on the gap between the two values, at least for some particular range of $n, r, s_{1}, \ldots, s_{l}$. It turns out that $G^{h}\left(n, r ; s_{1}, \ldots, s_{l}\right)$ and $G\left(n, r ; s_{1}, \ldots, s_{l}\right)$ have the same asymptotic behaviour, i.e. $s_{1}^{n+1} /(n+1) ! s_{2}^{n}$ (see Remark (4.10), (ii)). Then, by using Theorem (2.5), we will obtain a sort of lifting theorem which states that the varieties $V \in \mathcal{S}\left(n, r ; s_{1}, \ldots, s_{l}\right)$ of maximal geometric genus $G\left(n, r ; s_{1}, \ldots, s_{l}\right)$ must in fact lie on a flag such as

$$
V=V_{s_{1}}^{n} \subset V_{s_{2}}^{n+1} \subset \ldots \subset V_{s_{l}}^{n+l-1} \subset \mathbf{P}^{r},
$$

where $V_{s}^{j}$ denotes a subvariety of $\mathbf{P}^{r}$ of degree $s$ and dimension $j$ (see Corollary (4.8) for a precise statement). In other words

$$
G\left(n, r ; s_{1}, \ldots, s_{l}\right)=\max \left\{p_{g}(V): V \in \mathcal{F}\left(n, r ; s_{1}, \ldots, s_{l}\right)\right\},
$$

where $\mathcal{F}\left(n, r ; s_{1}, \ldots, s_{l}\right)$ denotes the set of all varieties $V_{s_{1}}^{n} \subset \mathbf{P}^{r}$ lying on a flag of type $(0.2)$. In the case of curves (i.e. $n=1$ ) and with respect to the arithmetic genus, the equality (0.3) is proved in [CCD2, Proposition (2.7) and Corollary (2.8)] for any $r$ and $s_{1}>>\ldots>>s_{l}$.

In the last Section 5 we deduce some new bounds for Castelnuovo's regularity of varieties verifying flag conditions, improving [NV, Corollary 15] in the case $l=$ $r-n=2$ and $s_{1}>>s_{2} \geq 3$, and extending [CCD2, Proposition (2.4)] to higher dimensional varieties (see Theorem (5.2) and (5.3)).

Finally we refer the reader to Remark (2.6) for a discussion on further generalizations of flag conditions (in particular we give a bound for the geometric genus of a variety in $\mathbf{P}^{r}$ which does not lie on any hypersurface of given degree), and to Remark (5.4) for a lower bound for the Euler characteristic of a surface in $\mathbf{P}^{4}$.

I'm grateful to L.Chiantini and C.Ciliberto for valuable discussions and suggestions, and their encouragement. I would like to thank V.Beorchia and A.Tortora for their bibliographic suggestions.

\section{Notation, COnVEntions And SOME BASIC DEFINitions}

(1.1) We work over an algebraically closed field $k$ of characteristic 0 and denote by $\mathbf{P}^{r}$ the projective space of dimension $r$ over the ground field $k$. Unless otherwise stated, a variety $V=V_{s}^{n} \subset \mathbf{P}^{r}$ is a reduced, irreducible, nondegenerate, possibly singular, projective scheme of dimension $n$ and degree $s$. Recall that a variety $V \subset \mathbf{P}^{r}$ is nondegenerate if it is not contained in a hyperplane. Given a variety $V=V_{s}^{n} \subset \mathbf{P}^{r}$, we denote by $\mathcal{O}_{V}$ its structure sheaf and by $\mathcal{I}_{V}$ the ideal sheaf of $V$ in $\mathbf{P}^{r}$. As usual, for any coherent sheaf $\mathcal{F}$ on $V$, we set $h^{i}(V, \mathcal{F})=\operatorname{dim}_{k} H^{i}(V, \mathcal{F})$. We denote by $V_{s}^{(i)}$ the intersection $V_{s}^{n} \cap L^{r-n+i}$, where $L^{r-n+i}(0 \leq i \leq n)$ is the generic $(r-n+i)$-dimensional subspace of $\mathbf{P}^{r}$. When there is no possibility of 
confusion, we use also $V^{(i)}$ to denote $V_{s}^{(i)}$, and sometimes the symbol $\Gamma$ to denote $V_{s}^{(0)}$. Notice that $V=V^{(n)}$.

(1.2) If $V=V_{s}^{n} \subset \mathbf{P}^{r}$ is a variety, for all $i=0, \ldots, n$ we denote by $p_{V^{(i)}}(t)\left(h_{V^{(i)}}(t)\right.$ resp.) the Hilbert polynomial (the Hilbert function resp.) of $V^{(i)}$. Moreover we indicate by $p_{g}(V)\left(p_{a}(V)\right.$ resp.) the geometric genus (the arithmetic genus resp.) of $V$. We denote by $\omega_{V}$ the dualizing sheaf of $V$ [Ht]. Notice that $h^{0}\left(V, \omega_{V}\right)=$ $h^{n}\left(V, \mathcal{O}_{V}\right)$ and so, when $n=1$, one has $h^{0}\left(V, \omega_{V}\right)=p_{a}(V)$. If $\widetilde{V} \rightarrow V$ is a resolution of singularities of $V$ then we have $p_{g}(V)=h^{0}\left(\widetilde{V}, \omega_{\widetilde{V}}\right)$ [Hr2]. We refer to $[\mathrm{R}]$ for the notion of variety with only canonical singularities. Next we recall that a variety $V=V_{s}^{n} \subset \mathbf{P}^{r}(n \geq 1)$ is said to be arithmetically Cohen-Macaulay (shortly a.C.M.) if all the restriction maps $H^{0}\left(\mathbf{P}^{r}, \mathcal{O}_{\mathbf{P}^{r}}(i)\right) \rightarrow H^{0}\left(V, \mathcal{O}_{V}(i)\right)(i \in \mathbf{Z})$ are surjective and $H^{j}\left(V, \mathcal{O}_{V}(i)\right)=0$ for all $i \in \mathbf{Z}$ and $1 \leq j \leq n-1$ [Se]. Observe that if $\operatorname{dim} V \geq 2$ then $V$ is a.C.M. if and only if its generic hyperplane section is. Also note that for an a.C.M. variety $V$ one has $h^{0}\left(V, \omega_{V}\right)=p_{a}(V)$. Finally we recall that a variety $V \subset \mathbf{P}^{r}$ is said to be $m$-regular (in the sense of Castelnuovo and Mumford) if $H^{i}\left(\mathbf{P}^{r}, \mathcal{I}_{V}(m-i)\right)=0$ for all $i>0(m \in \mathbf{Z})$. In such a case it is known that the homogenous ideal of $V$ is generated in degree $\leq m[\mathrm{M}]$.

(1.3) Throughout the paper we fix integers $n, r, s_{1}, \ldots, s_{l}$ such that $1 \leq n \leq r-2$, $2 \leq l \leq r-n$ and $s_{i} \geq r-n-i+2$ for all $i=1, \ldots, l$. We define the numbers $a_{i}$ and $b_{i}$ by dividing

$$
s_{i}-1=a_{i} s_{i+1}+b_{i}, \quad 0 \leq b_{i}<s_{i+1},
$$

for all $i=1, \ldots, l$, where $s_{l+1}:=r-n-l+1$. When $l \leq 3$, we will simplify this notation by setting $d=s_{1}, s=s_{2}$ and $t=s_{3}$ (see $\S 3, \S 4$ and also (1.5) below). We denote by $\mathcal{S}\left(n, r ; s_{1}, \ldots, s_{l}\right)$ the set of all varieties $V$ verifying a flag condition of type $\left(n, r ; s_{1}, \ldots, s_{l}\right)$ and define $\mathcal{F}\left(n, r ; s_{1}, \ldots, s_{l}\right)$ as the set of all varieties $V_{s_{1}}^{n} \subset \mathbf{P}^{r}$ lying on a flag such as $(0.2)$. Moreover we denote by $\widetilde{\mathcal{F}}\left(n, r ; s_{1}, \ldots, s_{l}\right)$ the set of all varieties $V_{s_{1}}^{n} \subset \mathbf{P}^{r}$ lying on flags of type $V=V_{s_{1}}^{n} \subset V_{s_{2}}^{n+1} \subset \ldots \subset V_{s_{l-1}}^{n+l-2} \subset \mathbf{P}^{r}$, where $V_{s_{l-1}}^{n+l-2} \subset \mathbf{P}^{r}$ is a variety not lying on varieties $W \subset \mathbf{P}^{r}$ of dimension $n+l-1$ and degree strictly less than $s_{l}$. Notice that $\mathcal{S}\left(n, r ; s_{1}, s_{2}\right)=\widetilde{\mathcal{F}}\left(n, r ; s_{1}, s_{2}\right)$.

(1.4) In order to make use of the results showed in [CCD2], which are essential in the rest of the present paper, and to get certain numerical estimates, we need the following assumptions on our numerical data $n, r, s_{1}, \ldots, s_{l}$ :

$$
\begin{gathered}
s_{i}>8(l-1)\left[(l-i+1)^{2}+2(l-i+1)+9\right] \frac{\left(s_{i+1}+1\right)^{3}}{r-n-i}, \\
s_{i}>\frac{\left(s_{i+1}+1\right)^{2}}{r-n-i}+(2(r-n)-2)\left(s_{i+1}+1\right), \\
s_{i}>2 \frac{\left(s_{i+1}+1\right)}{r-n-i} \prod_{j=1}^{r-n-i}\left[(r-n+1-i) !\left(s_{i+1}+1\right)\right]^{\frac{1}{r-n+1-i-j}}
\end{gathered}
$$

for all $i=1, \ldots, l-1$, and

$$
s_{1}>8 n !\left[4(l+1)^{n+1}+2^{n-1} n(n+8)\right] \frac{\left(s_{2}+1\right)^{3}}{(r-n-1)^{2}} .
$$


For instance, we need the hypothesis (1.4.1) to use [CCD2, Proposition (1.1)] for proving, with the assumption (1.4.4), our Propositions A and B (see $\S 2$ and the Appendix), and the hypotheses (1.4.2) and (1.4.3) to use [CCD2, Proposition (2.1)] for proving our main result, i.e. Theorem (2.5). We will abbreviate these assumptions by writing

$$
s_{1}>>\ldots>>s_{l} .
$$

As in [CCD2], these numerical hypotheses are certainly not the sharpest for our purposes. They are only of the simplest form we were able to conceive (however, in Section 3 we will simplify these assumptions).

(1.5) Now we define the function $G^{h}\left(n, r ; s_{1}, \ldots, s_{l}\right)$ which will represent our upper bound for $G\left(n, r ; s_{1}, \ldots, s_{l}\right)$. To do this, we simply need that $s_{i}>s_{i-1}^{2}-s_{i-1}$, for all $i=1, \ldots, l-1$. We put

$$
G^{h}\left(n, r ; s_{1}, \ldots, s_{l}\right):=\sum_{i=1}^{\infty}\left(\begin{array}{c}
i-1 \\
n-1
\end{array}\right)\left(s_{1}-h\left(r-n+1 ; s_{1}, \ldots, s_{l}\right)(i)\right)
$$

where the function $h\left(r-n+1 ; s_{1}, \ldots, s_{l}\right)$ is defined by induction on $l$ as follows. Put $r^{\prime}=r-n+1$. For $l=2$ we define

$$
h\left(r^{\prime} ; s_{1}, s_{2}\right)(i):=\sum_{j=0}^{i} \Delta h\left(r^{\prime} ; s_{1}, s_{2}\right)(j),
$$

where

$$
\Delta h\left(r^{\prime} ; s_{1}, s_{2}\right)(j):= \begin{cases}0, & j<0, \\ \left(r^{\prime}-2\right) j+1, & 0 \leq j \leq a_{2}, \\ s_{2}, & a_{2}<j \leq a_{1}, \\ s_{2}+k-\left(r^{\prime}-2\right)\left(j-a_{1}\right), & a_{1}<j \leq a_{1}+\delta \\ s_{2}+k-\left(r^{\prime}-2\right)\left(j-a_{1}\right)-1, & a_{1}+\delta<j \leq a_{1}+a_{2}+e, \\ 0, & j>a_{1}+a_{2}+e,\end{cases}
$$

with $k, \delta$ and $e$ defined as follows

$$
\begin{gathered}
b_{1}<a_{2}\left(r^{\prime}-1-b_{2}\right) \Rightarrow e=0, b_{1}=k a_{2}+\delta, 0 \leq \delta<a_{2}, \\
b_{1} \geq a_{2}\left(r^{\prime}-1-b_{2}\right) \Rightarrow e=1, b_{1}+r^{\prime}-2-b_{2}=k\left(a_{2}+1\right)+\delta, 0 \leq \delta<a_{2}+1 .
\end{gathered}
$$

Next, for $l \geq 3$, put

$$
\begin{aligned}
h\left(r^{\prime} ;\right. & \left.s_{1}, \ldots, s_{l}\right)(i) \\
& := \begin{cases}\sum_{j=0}^{i} h\left(r^{\prime}-1 ; s_{2}, \ldots, s_{l}\right)(j), & 0 \leq i \leq a_{1}, \\
\min \left\{s_{1}, h\left(r^{\prime} ; s_{1}, \ldots, s_{l}\right)\left(a_{1}\right)+h\left(r^{\prime} ; s_{1}, \ldots, s_{l}\right)\left(i-a_{1}\right)-1\right\}, & i>a_{1} .\end{cases}
\end{aligned}
$$

These functions $h\left(r^{\prime} ; s_{1}, \ldots, s_{l}\right)$ are introduced in [CCD2] (see also [CCD1] and [D] for the case $l=2$ ), to which we refer for a detailed discussion on their definition and properties.

We think it will be useful to briefly recall some basic facts, under the assumption $s_{1}>>\ldots>s_{l}$. First, $h\left(r ; s_{1}, \ldots, s_{l}\right)(i)=s_{1}$ for $i>>0$, and for any curve $V \in \mathcal{F}\left(1, r ; s_{1}, \ldots, s_{l}\right)$ one has $h_{\Gamma} \geq h\left(r ; s_{1}, \ldots, s_{l}\right)$ (recall that $\left.\Gamma=V^{(0)}\right)$. In other words $h\left(r ; s_{1}, \ldots, s_{l}\right)$ is a lower approximating function (LAF) for the set $\left\{h_{\Gamma}: V \in \mathcal{F}\left(1, r ; s_{1}, \ldots, s_{l}\right)\right\}$. In particular our definition (1.5.1) makes sense. 
By using the well known formula $p_{g}(V) \leq p_{a}(V) \leq \sum_{i=1}^{\infty}\left(s_{1}-h_{\Gamma}(i)\right)$ [EH], it follows that $G^{h}\left(1, r ; s_{1}, \ldots, s_{l}\right)$ is an upper bound for the numerical set $\left\{p_{a}(V)\right.$ : $\left.V \in \mathcal{F}\left(1, r ; s_{1}, \ldots, s_{l}\right)\right\}$. Actually $G^{h}\left(1, r ; s_{1}, \ldots, s_{l}\right)$ turns out to be an upper bound for the set $\left\{p_{a}(V): V \in \mathcal{S}\left(1, r ; s_{1}, \ldots, s_{l}\right)\right\}$, in particular for $G\left(1, r ; s_{1}, \ldots, s_{l}\right)$ (see $(0.1))$. Moreover one proves that in the special case $l=2$ the function $h\left(r ; s_{1}, s_{2}\right)$ is the sharp function (SF) for the set $\left\{h_{\Gamma}: V \in \mathcal{S}\left(1, r ; s_{1}, s_{2}\right)\right\}$, i.e. $h\left(r ; s_{1}, s_{2}\right)$ is an LAF for this set and there is an a.C.M. curve $V \in \mathcal{S}\left(1, r ; s_{1}, s_{2}\right)$ such that $h_{\Gamma}=h\left(r ; s_{1}, s_{2}\right)$. It follows that $G^{h}\left(1, r ; s_{1}, s_{2}\right)$ is a sharp upper bound for the set $\left\{p_{a}(V): V \in \mathcal{S}\left(1, r ; s_{1}, s_{2}\right)\right\}$. In particular observe that, for $s_{2}=r-1$ (for $r=3$ resp.), we find again a famous theorem by Castelnuovo [Ca], [EH] (by Halphen [Ha], [GP] resp.). On the other hand, when $l \geq 3$, one does not know whether $h\left(r ; s_{1}, \ldots, s_{l}\right)$ is an LAF for $\left\{h_{\Gamma}: V \in \mathcal{S}\left(1, r ; s_{1}, \ldots, s_{l}\right)\right\}$, and one can show that $h\left(r ; s_{1}, \ldots, s_{l}\right)$ is not an SF for the set $\left\{h_{\Gamma}: V \in \mathcal{F}\left(1, r ; s_{1}, \ldots, s_{l}\right)\right\}$. In particular, when $l \geq 3, G^{h}\left(1, r ; s_{1}, \ldots, s_{l}\right) \neq G\left(1, r ; s_{1}, \ldots, s_{l}\right)$ [CCD2, Remark (2.5)].

However in [CCD3] the authors refine the function $h\left(4 ; s_{1}, s_{2}, s_{3}\right)$ and determine, in the special case $r=4$ and $l=3$, the sharp function $\tilde{h}\left(4 ; s_{1}, s_{2}, s_{3}\right)$ for the set $\left\{h_{\Gamma}: V \in \mathcal{F}\left(1,4 ; s_{1}, s_{2}, s_{3}\right)\right\}$, which we are going to define.

To simplify the notation, set $d=s_{1}, s=s_{2}, t=s_{3}, m=a_{1}, \epsilon=b_{1}, \alpha=a_{2}$, $\beta=b_{2}$ (compare with (1.3)). As usual assume $d>>s>>t$. Next,

if $\epsilon \geq s-(\beta+1)(\alpha+\beta+2-t)$, divide $s-\epsilon-1=u(\alpha+\beta+2-t)+v$ and put $a=t-u-1$ and $b=m+\alpha+\beta+1-u-v$;

if $\epsilon<s-(\beta+1)(\alpha+\beta+2-t)$, divide $\epsilon=u(\alpha+\beta+1)+v$ and put $a=u$ and $b=m+u+v+1$.

Then divide $s+a=p t+q, 0 \leq q<t$, and put $F_{d, s, t}^{\prime}=F^{\prime}$ defined by

$$
F^{\prime}(n):=\left\{\begin{array}{lll}
\Delta h(4 ; d, s, t)(n), & \text { if } \quad n \leq m, \\
s-\left(\begin{array}{c}
n-m+1 \\
2
\end{array}\right), & \text { if } \quad m \leq n \leq m+a, \\
s-\left(\begin{array}{c}
n-m+1 \\
2
\end{array}\right)-(n-m-a), & \text { if } \quad m+a \leq n \leq m+t-1, \\
s-\left(\begin{array}{c}
t \\
2
\end{array}\right)-(t-a-1)-t(n-m-t+1), & \text { if } \quad m+t-1 \leq n \leq m+p, \\
\left(\begin{array}{c}
t+p+m-n-1 \\
2
\end{array}\right)+(m+p+q-n), & \text { if } \quad m+p \leq n \leq m+p+q, \\
\left(\begin{array}{c}
t+p+m-n-1 \\
2
\end{array}\right), & \text { if } n \geq m+p+q .
\end{array}\right.
$$

Finally put

$$
F_{d, s, t}(n):= \begin{cases}F^{\prime}(n) & \text { if } n<b \quad \text { or } n \geq m+p+q \\ F^{\prime}(n)-1 & \text { if } \quad b \leq n \leq m+p+q-1\end{cases}
$$

and define

$$
\tilde{h}(4 ; d, s, t)(j):=\sum_{i=0}^{j} F_{d, s, t}(i)
$$

and

$$
G^{\tilde{h}}(1,4 ; d, s, t):=\sum_{i=1}^{\infty}(d-\tilde{h}(4 ; d, s, t)(i)) .
$$

It turns out that $G^{\tilde{h}}(1,4 ; d, s, t)$ is a sharp upper bound for the set $\left\{p_{a}(V): V \in\right.$ $\mathcal{S}(1,4 ; d, s, t)\}$ [CCD3]. In Section 4 we will use these new functions $\tilde{h}(4 ; d, s, t)$ to refine our bound $G^{h}\left(n, r ; s_{1}, \ldots, s_{r-n}\right)$, in the particular case $l=r-n \geq 3$. 


\section{THE MAIN THEOREM}

In this section we prove our main result on the bound of the geometric genus of a variety verifying flag conditions (i.e. Theorem (2.5)). First of all we need some numerical properties of the functions $G^{h}\left(n, r ; s_{1}, \ldots, s_{l}\right)$, which will be essential in the rest of the paper (see Propositions A and B below, whose proof we exhibit at the end of the article, in the Appendix). Next we need some set-theoretical properties concerning the sets defined in (1.3) (see Lemma (2.1)) and then a preliminary proposition (i.e. Proposition (2.4)), which can be seen as an extension of [CCD2, Proposition (2.1)] and [D, Theorem (1.1)]. Finally, in Remark (2.6), we make some comments on varieties verifying generalized flag conditions. We keep all the notation and conventions we introduced in $\S 1$.

Proposition A. With the above notation, assume that

$$
s_{1}>>s_{2}>>\ldots>>s_{l} \text {. }
$$

Then there are rational numbers $\eta$ and $O$ such that

$$
|\eta| \leq \frac{3}{4}, \quad|O| \leq s_{1}^{n-1} \frac{s_{2}^{4-n}}{s_{3}^{2}}\left[4(l+1)^{n+1}+2^{n-1} n(n+8)\right]
$$

and that

$$
\begin{gathered}
G^{h}\left(n, r ; s_{1}, \ldots, s_{l}\right)=\frac{s_{1}{ }^{n+1}}{(n+1) ! s_{2}{ }^{n}} \\
+\frac{s_{1}{ }^{n}}{2 n ! s_{2}{ }^{n-1}}\left[\frac{s_{2}}{s_{3}}+\ldots+\frac{s_{l}}{s_{l+1}}-l-\frac{2}{r-n+1-l}+\eta-(n-1)\right]+O .
\end{gathered}
$$

As a consequence of the previous proposition, one obtains some properties of monotonicity of the functions $G^{h}\left(n, r ; s_{1}, \ldots, s_{l}\right)$.

Proposition B. With the above notation, assume that

$$
s_{1}>>s_{2}>>\ldots>s_{l} \text {. }
$$

Put

$$
\delta_{2}:=\frac{s_{1}^{n}}{2 n ! s_{2}{ }^{n-1}}\left[\frac{2 s_{1}}{(n+1) s_{2}\left(s_{2}+1\right)}-(l+n+4)\right]
$$

and, for $i=3, \ldots, l$,

$$
\delta_{i}:=\frac{s_{1}^{n}}{2 n ! s_{2}{ }^{n-1}}\left[\frac{s_{i-1}}{s_{i}\left(s_{i}+1\right)}-3 \frac{s_{i}}{r-n+1-i}\right] .
$$

Then we have

(i) $0<\delta_{l}<\delta_{l-1}<\ldots<\delta_{3}<\delta_{2}$;

(ii) $\delta_{i} \leq G^{h}\left(n, r ; s_{1}, \ldots, s_{l}\right)-G^{h}\left(n, r ; s_{1}, \ldots, s_{i-1}, s_{i}+1\right)$, for all $i=2, \ldots, l$.

As we said, we refer to the Appendix for the proofs of Propositions A and B.

Lemma 2.1. As usual assume that $s_{1}>>\ldots>>s_{l}$. Then the following properties hold.

(1) $\mathcal{F}\left(n, r ; s_{1}, s_{2}, \ldots, s_{l}\right) \subset \widetilde{\mathcal{F}}\left(n, r ; s_{1}, s_{2}, \ldots, s_{l}\right) \subset \mathcal{S}\left(n, r ; s_{1}, s_{2}, \ldots, s_{l}\right)$.

(2) $\mathcal{S}\left(n, r ; s_{1}, s_{2}, \ldots, s_{l}\right) \subset \bigcup_{i=2}^{l} \widetilde{\mathcal{F}}\left(n, r ; s_{1}, s_{2}, \ldots, s_{i}+1\right) \cup \mathcal{F}\left(n, r ; s_{1}, s_{2}, \ldots, s_{l}\right)$.

(3) If $V \in \mathcal{S}\left(n, r ; s_{1}, s_{2}, \ldots, s_{l}\right)$ then, for all $j=1, \ldots, n$, one has

$$
V^{(j)} \in \bigcup_{i=2}^{l} \tilde{\mathcal{F}}\left(j, r-n+j ; s_{1}, s_{2}, \ldots, s_{i}+1\right) \cup \mathcal{F}\left(j, r-n+j ; s_{1}, s_{2}, \ldots, s_{l}\right) .
$$


(4) If $V \in \widetilde{\mathcal{F}}\left(n, r ; s_{1}, s_{2}, \ldots, s_{l}\right)$ then, for all $j=1, \ldots, n$, one has

$$
V^{(j)} \in \widetilde{\mathcal{F}}\left(j, r-n+j ; s_{1}, s_{2}, \ldots, s_{l}\right) .
$$

Proof. Property (1) follows by using Bezout's theorem, taking into account our numerical hypotheses $s_{1}>>\ldots>>s_{l}$.

To prove (2), consider a variety $V_{s_{1}}^{n} \in \mathcal{S}\left(n, r ; s_{1}, s_{2}, \ldots, s_{l}\right)$ and notice that if $V_{s_{1}}^{n}$ is not contained in any $V_{\sigma}^{n+1}$ with $\sigma<s_{2}+1$ then $V_{s_{1}}^{n} \in \widetilde{\mathcal{F}}\left(n, r ; s_{1}, s_{2}+1\right)$. Otherwise $V_{s_{1}}^{n}$ is contained in some variety $V_{s_{2}}^{n+1}$. If this variety is not contained in any $V_{\sigma}^{n+2}$ with $\sigma<s_{3}+1$ then $V_{s_{1}}^{n} \in \widetilde{\mathcal{F}}\left(n, r ; s_{1}, s_{2}, s_{3}+1\right)$. Otherwise $V_{s_{2}}^{n+1}$ is contained in some variety $V_{s_{3}}^{n+2}$. Continuing in this fashion one shows property (2).

In order to prove property (3), notice that by the lifting theorem $[\mathrm{CC}$, Theorem (0.2)], the generic linear section $V_{s_{1}}^{(j)}=V_{s_{1}}^{n} \cap L^{r-n+j}$ cannot lie on varieties of type $W_{\sigma}^{j+1} \subset L^{r-n+j}$ with $\sigma<s_{2}$. Hence, as before, we have two possibilties. If $V_{s_{1}}^{(j)}$ is not contained in any $W_{\sigma}^{j+1} \subset L^{r-n+j}$ with $\sigma<s_{2}+1$ then $V_{s_{1}}^{(j)} \in$ $\widetilde{\mathcal{F}}\left(j, r-n+j ; s_{1}, s_{2}+1\right)$. Otherwise $V_{s_{1}}^{(j)}$ is contained in some $W_{s_{2}}^{j+1} \subset L^{r-n+j}$ which can be lifted, by the quoted theorem, to a $V_{s_{2}}^{n+1} \subset \mathbf{P}^{r}$ containing $V_{s_{1}}^{n}$ and such that $V_{s_{2}}^{(j+1)}=W_{s_{2}}^{j+1}$. From this we deduce, again by using the lifting theorem, that $W_{s_{2}}^{j+1}$ is not contained in any $W_{\sigma}^{j+2} \subset L^{r-n+j}$ with $\sigma<s_{3}$. If $W_{s_{2}}^{j+1}$ is not contained in any $W_{\sigma}^{j+2} \subset L^{r-n+j}$ with $\sigma<s_{3}+1$ then we have $V_{s_{1}}^{(j)} \in$ $\widetilde{\mathcal{F}}\left(j, r-n+j ; s_{1}, s_{2}, s_{3}+1\right)$. Otherwise $W_{s_{2}}^{j+1}$ is contained in some $W_{s_{3}}^{j+2}$. Continuing in the same manner one shows that either

$$
V^{(j)} \in \bigcup_{i=2}^{l} \mathcal{S}\left(j, r-n+j ; s_{1}, s_{2}, \ldots, s_{i}+1\right)
$$

or

$$
V^{(j)} \in \mathcal{F}\left(j, r-n+j ; s_{1}, s_{2}, \ldots, s_{l}\right)
$$

i.e. property (3).

Finally we notice that (4) follows again by using [CC, Theorem (0.2)].

The next lemma can be seen as a generalization of a classical result (i.e. [EH, Corollary (3.2)]) and, together with Remark (2.3) below, it will be essential in the rest of the paper.

Lemma 2.2. Let $V \subset \mathbf{P}^{r}$ be a variety of dimension $n$ and degree $d$. Then one has

$$
p_{g}(V) \leq h^{0}\left(V, \omega_{V}\right) \leq \sum_{i=1}^{\infty}\left(\begin{array}{c}
i-1 \\
n-1
\end{array}\right)\left(d-h_{\Gamma}(i)\right),
$$

where $\Gamma=V^{(0)}$.

Proof. By [Ci2, proof of Lemma (2.3.1)], we have that $p_{g}(V) \leq h^{0}\left(V, \omega_{V}\right)$. On the other hand, by the definition of dualizing sheaf [Ht] one has $h^{0}\left(V, \omega_{V}\right)=h^{n}\left(V, \mathcal{O}_{V}\right)$. And so, in order to prove our claim, it suffices to show that

$$
h^{n}\left(V, \mathcal{O}_{V}\right) \leq \sum_{i=1}^{\infty}\left(\begin{array}{c}
i-1 \\
n-1
\end{array}\right)\left(d-h_{\Gamma}(i)\right) .
$$

This is an application of the classical method of Castelnuovo, for which we refer to [NV, Lemma 5] (compare with [Hr2] and [D, Proposition (1.2)]). 
Remark 2.3. Notice that, by the proof of previous lemma, it follows that

$$
V \quad \text { a.C.M. } \Longrightarrow \quad h^{0}\left(V, \omega_{V}\right)=\sum_{i=1}^{\infty}\left(\begin{array}{c}
i-1 \\
n-1
\end{array}\right)\left(d-h_{\Gamma}(i)\right) \text {. }
$$

It is well known that, in the case of curves (i.e. $n=1$ ), the converse of (2.3.1) holds $[\mathrm{EH}]$. It is no longer true for higher dimensional varieties. For instance, consider the Veronese surface $S$ in $\mathbf{P}^{4}$, which is a smooth surface not a.C.M., being a projection of the Veronese surface in $\mathbf{P}^{5}$. However, by direct computation, one sees that

$$
p_{a}(S)=p_{g}(S)=\sum_{i=1}^{\infty}(i-1)\left(4-h_{\Gamma}(i)\right)=0 .
$$

It may be interesting to point out that the Veronese surface is the only example in $\mathbf{P}^{4}$ for which the converse of (2.3.1) fails, in the following sense.

For a smooth surface $V$ of degree $d$ one has $p_{a}(V) \leq p_{g}(V)$, and so, by the previous lemma, $p_{a}(V) \leq \sum_{i=1}^{\infty}(i-1)\left(d-h_{\Gamma}(i)\right)$. Combining the classical result of Severi $[\mathrm{Sv}]$ that the Veronese surface is the only smooth surface in $\mathbf{P}^{4}$ which is not linearly normal, with [D, Theorem (3.7) and proof], one can see that if $V$ is a projective, nondegenerate, irreducible and smooth surface in $\mathbf{P}^{4}$, of degree $d$, then $p_{a}(V)=\sum_{i=1}^{\infty}(i-1)\left(d-h_{\Gamma}(i)\right)$ if and only if $V$ is a.C.M. or the Veronese surface.

Proposition 2.4. Let $l \geq 2$ be an integer and let us consider a flag

$$
V:=V_{s_{1}}^{n} \subset V_{s_{2}}^{n+1} \subset \ldots \subset V_{s_{l-1}}^{n+l-2}
$$

of projective, irreducible, nondegenerate varieties in $\mathbf{P}^{r}$. Let us assume that $V_{s_{l-1}}^{n+l-2}$ is not contained in any $V_{s}^{n+l-1}$ with $s<s_{l}$. Consider the generic $(r-n)$ dimensional subspace $L^{r-n}$ of $\mathbf{P}^{r}$ and put $\Gamma:=V \cap L^{r-n}$. As usual, assume $s_{1}>>\ldots>>s_{l}$. Then

$$
h_{\Gamma} \geq h\left(r-n+1 ; s_{1}, \ldots, s_{l}\right) .
$$

In particular, one has

$$
p_{g}(V) \leq G^{h}\left(n, r ; s_{1}, \ldots, s_{l}\right) .
$$

Proof. Since $V \in \widetilde{\mathcal{F}}\left(n, r ; s_{1}, s_{2}, \ldots, s_{l}\right)$ then, by Lemma (2.1), (4), one has $V^{(1)} \in$ $\widetilde{\mathcal{F}}\left(1, r-n+1 ; s_{1}, s_{2}, \ldots, s_{l}\right)$. Hence we can apply [CCD2, Proposition (2.1)] to the curve $V^{(1)}$ and deduce that $h_{\Gamma} \geq h\left(r-n+1 ; s_{1}, \ldots, s_{l}\right)$.

The last assertion now follows by Lemma (2.2) and by the definition (1.5.1) of the function $G^{h}\left(n, r ; s_{1}, \ldots, s_{l}\right)$.

Now we are in position to prove our main result. First we recall that the number $\delta_{l}$, which appears in its statement, was defined in the statement of Proposition B.

Theorem 2.5. Let $V$ be an irreducible nondegenerate variety of dimension $n$, degree $s_{1}$ and geometric genus $p_{g}(V)$ in $\mathbf{P}^{r}$. Let $l=2, \ldots, r-1$ be an integer and let $s_{2}, \ldots, s_{l}$ be positive integers (with $s_{l} \geq r-n-l+2$ ) such that, as usual,

$$
s_{1}>>s_{2}>>\ldots>>s_{l} \text {. }
$$

Let us also assume that for all $i=2, \ldots, l$, there is no $V_{s}^{n+i-1}$ containing $V$ with $s<s_{i}$. Then we have:

(i) $p_{g}(V) \leq G^{h}\left(n, r ; s_{1}, \ldots, s_{l}\right)$; 
(ii) if $G^{h}\left(n, r ; s_{1}, \ldots, s_{l}\right)-p_{g}(V)<\delta_{l}$, there is a unique flag

$$
V:=V_{s_{1}}^{n} \subset V_{s_{2}}^{n+1} \subset \ldots \subset V_{s_{l}}^{n+l-1} \subset \mathbf{P}^{r} .
$$

Proof. (i) Since $V \in \mathcal{S}\left(n, r ; s_{1}, s_{2}, \ldots, s_{l}\right)$ then, by using Lemma (2.1), (2), either $V \in \widetilde{\mathcal{F}}\left(n, r ; s_{1}, s_{2}, \ldots, s_{i}+1\right)$ for some $i=2, \ldots, l$ or $V \in \mathcal{F}\left(n, r ; s_{1}, s_{2}, \ldots, s_{l}\right)$. In the first case, by Proposition (2.4), we have $p_{g}(V) \leq G^{h}\left(n, r ; s_{1}, s_{2}, \ldots, s_{i}+1\right)$, which is strictly less than $G^{h}\left(n, r ; s_{1}, s_{2}, \ldots, s_{l}\right)$ by Proposition B. Otherwise $V \in$ $\mathcal{F}\left(n, r ; s_{1}, s_{2}, \ldots, s_{l}\right)$. In particular, by Lemma $(2.1),(1), V \in \widetilde{\mathcal{F}}\left(n, r ; s_{1}, s_{2}, \ldots, s_{l}\right)$ and so, again by Proposition $(2.4), p_{g}(V) \leq G^{h}\left(n, r ; s_{1}, s_{2}, \ldots, s_{l}\right)$.

(ii) As before, by using Lemma (2.1), (2), we have, a priori, two cases. If $V \in$ $\widetilde{\mathcal{F}}\left(n, r ; s_{1}, s_{2}, \ldots, s_{i}+1\right)$ for some $i=2, \ldots, l$ then, by Proposition (2.4), we have $p_{g}(V) \leq G^{h}\left(n, r ; s_{1}, s_{2}, \ldots, s_{i}+1\right)$ and so, by Proposition B,

$$
\begin{aligned}
& G^{h}\left(n, r ; s_{1}, s_{2}, \ldots, s_{l}\right)-p_{g}(V) \\
& \quad \geq G^{h}\left(n, r ; s_{1}, s_{2}, \ldots, s_{l}\right)-G^{h}\left(n, r ; s_{1}, s_{2}, \ldots, s_{i}+1\right) \geq \delta_{i} \geq \delta_{l},
\end{aligned}
$$

which is in contrast with our hypotheses. It follows that $V \in \mathcal{F}\left(n, r ; s_{1}, s_{2}, \ldots, s_{l}\right)$, i.e. $V$ lies on a flag whose uniqueness follows by Bezout's theorem and by the hypotheses on the $s_{i}$ 's.

Remark 2.6. As in [CCD2, Remark (2.9)], we notice that the flag conditions we considered in the present paper could be generalized. Precisely, one could say that a variety $V \subset \mathbf{P}^{r}$ verifies a generalized flag condition of type $\left(n, r ; s_{1}, s_{2}^{\left(i_{2}\right)}, \ldots, s_{l}^{\left(i_{l}\right)}\right)$ if $V$ is of dimension $n$ and degree $s_{1}$ and does not lie on any variety $V_{s}^{i_{j}}$ with $s<s_{j}$, $j=2, \ldots, l$. Then one could try to determine or estimate the maximum geometric genus of varieties verifying such a generalized flag condition. We are not able to deal with this problem in such generality. However we want to briefly discuss a particular instance of it, for which we are able to provide some information and give some flavour of what the general answer should be.

Precisely, we consider a flag condition of type $\left(n, r ; d, t^{(r-1)}\right)$, i.e. we would like to estimate the geometric genus $p_{g}(V)$ of a variety $V \subset \mathbf{P}^{r}$ of degree $d$ not lying on any hypersurface of degree $<t$. Such an estimate is not difficult to find. In fact, let $\sigma(n+1, r ; t)$ be the maximum integer such that any variety $W^{n+1} \subset \mathbf{P}^{r}$ of degree $<\sigma(n+1, r ; t)$ in $\mathbf{P}^{r}$ lies on a hypersurface of degree $t-1$. Then, if $d>>0$, by our Theorem (2.5), we have

$$
p_{g}(V) \leq G^{h}(n, r ; d, \sigma(n+1, r ; t))
$$

Of course the problem remains of determining $\sigma(n+1, r ; t)$. It is not so difficult to give an estimate of $\sigma(n+1, r ; t)$ :

$$
\sigma(n+1, r ; t)>\max \left\{s \in \mathbf{N}: s\left(\begin{array}{c}
t-1+n \\
n+1
\end{array}\right)+\left(\begin{array}{c}
t-1+n \\
n
\end{array}\right)<h^{0}\left(\mathbf{P}^{r}, \mathcal{O}_{\mathbf{P}^{r}}(t-1)\right)\right\}
$$

Indeed one verifies that for every variety $W^{n+1}$ of degree $s$ in $\mathbf{P}^{r}$ one has

$$
h_{W^{n+1}}(i) \leq s\left(\begin{array}{c}
i+n \\
n+1
\end{array}\right)+\left(\begin{array}{c}
i+n \\
n
\end{array}\right) \text {. }
$$


In conclusion, if we put $\tilde{\sigma}:=\tilde{\sigma}(n+1, r, t):=\frac{(n+1) !}{r !} \frac{1}{t-1}(t-1+r) \ldots(t+n)-\frac{n+1}{t-1}$, one finds with the above estimate (by Proposition A and Theorem (2.5))

$$
p_{g}(V) \leq \frac{d^{n+1}}{(n+1) ! \tilde{\sigma}^{n}}+O\left(d^{n}\right),
$$

from which we deduce, when $t \geq n+1$,

$$
p_{g}(V) \leq \frac{(r !)^{n} d^{n+1}}{(n+1) !^{n+1} t^{n(r-n-1)}}+O\left(d^{n}\right)
$$

and we remark that, using a variety $W^{n+1}$ given as a complete intersection of $r-n-1$ generic hypersurfaces of degree $t$ in $\mathbf{P}^{r}$, and taking hypersurface sections of $W^{n+1}$ of very high degree, we can find a variety $V^{n}$ verifying the desired flag condition, whose geometric genus is

$$
p_{g}(V)=\frac{d^{n+1}}{(n+1) ! t^{n(r-n-1)}}+O\left(d^{n}\right)
$$

(to obtain (2.6.2) use a similar argument as in Lemma (4.7) of Section 4 below).

Notice that by (2.6.1) and (2.6.2) one can deduce that if $d>>0, t^{r-n-1} \geq n+1$ and $t^{r-n-1}$ divides $d$, then any variety $V_{d}^{n} \subset \mathbf{P}^{r}$ not contained in hypersurfaces of degree $<t$ and of maximal geometric genus, must lie on a hypersurface of degree $<\tau$ where $\tau:=\min \left\{i: \frac{(n+1) !}{r !} \frac{1}{i-1}(i-1+r) \ldots(i+n)-\frac{n+1}{i-1}>t^{r-n-1}\right\}$. In particular $V_{d}^{n}$ is contained in some hypersurface of degree

$$
\leq t\left[\sqrt[r-n-1]{\frac{r !}{(n+1) !}}\right]
$$

For instance we have that if 9 divides $d$ and $d>>0$, then any curve $C_{d} \subset \mathbf{P}^{4}$ not contained in quadrics and of maximal geometric genus must lie on a sextic, or also that any surface $S_{d} \subset \mathbf{P}^{5}$ not contained in quadrics and of maximal geometric genus must lie on a hypersurface of degree 8 . Notice that in the previous examples one expects that the curve $C_{d}$ (resp. the surface $S_{d}$ ) lies on some cubic.

\section{The CASe of CODimension two}

In this section we analyse the case $l=r-n=2$, but first we want to make some comments on the case $l=2, r-n \geq 2$. To simplify the notation, we put $d=s_{1}$, $s=s_{2}, m=a_{1}, \epsilon=b_{1}, w=a_{2}$ and $v=b_{2}$ (compare with (1.3)).

As we said in (1.5), in the case of curves, i.e. when $n=1$, the number $G^{h}(1, r ; d, s)$ is actually an upper bound for the arithmetic genus of curves belonging to $\mathcal{S}(1, r ; d, s)$ and in fact it is sharp for any $d>>s \geq r-1$ [CCD1]. But, with respect to the geometric genus, it is only known that the bound $G^{h}(1, r ; d, s)$ is sharp for certain ranges of $r, d$ and $s$. For instance, this is the case when $s=r-1$ [EH], or $r=3$ and $d>s^{2}-s$ [GP], or any $r, d>>s \geq 2(r-2), 0<v<r-2$ and $\epsilon \geq w(r-n-1-v)[\mathrm{CCD} 1]$.

Of course, the question of sharpness in the case $n \geq 2$ is more difficult. Examples of varieties verifying flag conditions of type $(n, r ; d, s)$ of maximal geometric genus $G^{h}(1, r ; d, s)$ can be found in [Hr2] for $s=r-n$, the so called Castelnuovo varieties, and in [D] for $r \geq 2 n+3, d>>s \geq(n+1)(r-n-1), 0<v<r-n-1$, $\epsilon \geq w(r-n-1-v)$.

All this investigation goes back to the first result in this direction, i.e. Halphen's theorem ([Ha], [GP]), which establishes the existence of the bound $G^{h}(1,3 ; d, s)$ and 
classifies the space curves whose geometric genus reaches the maximum. It turns out that, for any $d>s^{2}-s$ and $C \in \mathcal{S}(1,3 ; d, s)$, one has $p_{g}(C) \leq G^{h}(1,3 ; d, s)$ and, when $C$ is smooth, $p_{g}(C)=G^{h}(1,3 ; d, s)$ if and only if $C$ is linked to a plane curve by a complete intersection of type $(s, m+1)$. In particular, such curves exist and are a.C.M..

The aim of this section is to extend Halphen's theorem to the case of codimension two (see Theorem (3.1) below). We will later use this result for constructing, at least in some range for the $s_{i}$ 's, varieties $V \in \mathcal{S}\left(n, r ; s_{1}, \ldots, s_{l}\right)$ with geometric genus sufficiently near to $G^{h}\left(n, r ; s_{1}, \ldots, s_{l}\right)$.

Theorem 3.1. Let $V \subset \mathbf{P}^{n+2}$ be a variety of degree $d$ and dimension $n$. Assume that $V$ is not contained in any hypersurface of degree $<s$, with $d>s^{2}-s, s \geq 2$. Put $d-1=m s+\epsilon, 0 \leq \epsilon<s$. Then one has:

(i) $p_{g}(V) \leq G^{h}(n, n+2 ; d, s)$.

(ii) If $V$ is linked to a complete intersection $V^{\prime} \subset \mathbf{P}^{n+2}$ of type $(1, s-1-\epsilon)$ by a complete intersection of two hypersurfaces of degrees $s$ and $m+1$, and if $p_{g}(V)=$ $h^{0}\left(V, \omega_{V}\right)$, then $p_{g}(V)=G^{h}(n, n+2 ; d, s)$. Moreover such varieties exist. And so the upper bound $G^{h}(n, n+2 ; d, s)$ is sharp, i.e. $G(n, n+2 ; d, s)=G^{h}(n, n+2 ; d, s)$, and, for $1 \leq n \leq 3$, it is attained by smooth varieties.

(iii) Assume $p_{g}(V)=G^{h}(n, n+2 ; d, s)$. Then $p_{g}(V)=h^{0}\left(V, \omega_{V}\right)$ and, when $d>\max \left\{4 s^{2},(n+s)^{2}\right\}, V$ is contained in a hypersurface of degree $s$ (i.e. $V \in$ $\mathcal{F}(n, n+2 ; d, s))$. Moreover, if $s=2$ or $\epsilon \notin\{0,1, s-3\}$, then $V$ is linked to a complete intersection $V^{\prime} \subset \mathbf{P}^{n+2}$ of type $(1, s-1-\epsilon)$ by a complete intersection of two hypersurfaces of degrees $s$ and $m+1$. In particular, $V$ is a Cohen-Macaulay and a.C.M. variety, and so, when $n \geq 4$ and $\epsilon \neq s-1, V$ must be singular.

Proof. First observe that if $s=2$ then our theorem is known by Harris' classification of Castelnuovo varieties [Hr2]. Hence we may assume $s>2$.

When $d>>s$, property (i) follows by our Theorem (2.5). However, in case of codimension two, by using [GP] and the generalized Laudal's lemma [St], we have that $h_{\Gamma} \geq h(3 ; d, s)$ also when $d>s^{2}-s$, and so, by Lemma (2.2), property (i) holds also under the more general hypotheses $d>s^{2}-s$.

In order to show (ii), first assume that $V$ is linked to a degenerate $V^{\prime} \subset \mathbf{P}^{n+2}$ and that $p_{g}(V)=h^{0}\left(V, \omega_{V}\right)$. Take the generic curve section $V^{(1)} \subset \mathbf{P}^{3}$. It is well know that $V^{(1)}$ is a.C.M. and of maximal arithmetic genus with respect to the flag condition $(1,3 ; d, s)[\mathrm{GP}]$. Hence $V$ is a.C.M. and $h_{\Gamma}(i)=h(3 ; d, s)(i)$ for all $i$. By Remark (2.3) and our hypotheses $p_{g}(V)=h^{0}\left(V, \omega_{V}\right)$, it follows that $p_{g}(V)=G^{h}(n, n+2 ; d, s)$.

Now, to prove the sharpness of the bound, consider a variety $V$ linked to a generic complete intersection $V^{\prime} \subset \mathbf{P}^{n+2}$ of type $(1, s-1-\epsilon)$, by a complete intersection of two generic hypersurfaces $F$ and $G$ containing $V^{\prime}$, of degree respectively $s$ and $m+1$ (by [PS] we know that, for $1 \leq n \leq 3, V$ is smooth). In order to evaluate the geometric genus of $V$, consider the blow-up $p: \widetilde{\mathbf{P}} \longrightarrow \mathbf{P}^{n+2}$ of $\mathbf{P}^{n+2}$ at $V^{\prime}$. Let $E$ be the exceptional divisor of the blow-up, and $\widetilde{F}=p^{*} F-E, \widetilde{G}=p^{*} G-E$ and $\widetilde{V}$ the proper transforms of $F, G$ and $V$ in $\widetilde{\mathbf{P}}$. Since $F$ and $G$ are generic with respect to the condition of containing $V^{\prime}$, then $\tilde{\widetilde{V}}=\widetilde{F} \cap \widetilde{G}, \widetilde{V}$ is smooth and so $p_{g}(V)=h^{0}\left(\widetilde{V}, \omega_{\widetilde{V}}\right)$. Taking into account that $\omega_{\widetilde{\mathbf{P}}}=\mathcal{O}_{\widetilde{\mathbf{P}}}(E)(-n-3)$, and using the adjunction formula, one sees that $\omega_{\widetilde{V}}=\left.\left(\mathcal{O}_{\widetilde{\mathbf{P}}}(-E)(m+s-n-2)\right)\right|_{\widetilde{V}}$. It follows that

$$
p_{g}(V)=h^{0}\left(\tilde{V}, \omega_{\tilde{V}}\right) \geq h^{0}\left(\mathbf{P}^{n+2}, \mathcal{I}_{V^{\prime}}(\nu)\right)-h^{0}\left(\mathbf{P}^{n+2}, \mathcal{I}_{M}(\nu)\right)
$$


where $M$ denotes the complete intersection $F \cap G$ and $\nu=m+s-n-2$. Let $\pi$ be a generic plane of $\mathbf{P}^{n+2}$, and put $\Gamma^{\prime}=\pi \cap V^{\prime}$ and $\bar{\Gamma}=\pi \cap M$. Using [D, Lemma (2.4) and proof] and taking into account that $V, V^{\prime}$ and $M$ are a.C.M., one sees that

$$
h^{0}\left(\mathbf{P}^{n+2}, \mathcal{I}_{V^{\prime}}(\nu)\right)-h^{0}\left(\mathbf{P}^{n+2}, \mathcal{I}_{M}(\nu)\right)=\sum_{i=0}^{\nu}\left(\begin{array}{c}
m+s-3-i \\
n-1
\end{array}\right)\left(h_{\bar{\Gamma}}(i)-h_{\Gamma^{\prime}}(i)\right) .
$$

By direct computation one has, for all $i$,

$$
h_{\bar{\Gamma}}(i)-h_{\Gamma^{\prime}}(i)=d-h(3 ; d, s)(m+s-2-i),
$$

from which we deduce that

$$
\begin{aligned}
& \sum_{i=0}^{\nu}\left(\begin{array}{c}
m+s-3-i \\
n-1
\end{array}\right)\left(h_{\bar{\Gamma}}(i)-h_{\Gamma^{\prime}}(i)\right) \\
& \quad=\sum_{j=n}^{m+s-2}\left(\begin{array}{l}
j-1 \\
n-1
\end{array}\right)(d-h(3 ; d, s)(j))=G^{h}(n, n+2 ; d, s) .
\end{aligned}
$$

Hence by (3.1.1) and (i) we get $p_{g}(V)=G^{h}(n, n+2 ; d, s)$, and so the proof of (ii) is complete.

In order to prove (iii), assume $p_{g}(V)=G^{h}(n, n+2 ; d, s)$ and notice that $p_{g}(V)=$ $h^{0}\left(V, \omega_{V}\right)$ by Lemma (2.2) and Proposition (2.4), which, as we said before, in codimension two, holds for $d>s^{2}-s$. Next note that, when $d>\max \left\{4 s^{2},(n+s)^{2}\right\}$, $V$ is contained in a hypersurface of degree $s$ by [D, Theorem (3.3)]. Since $V$ has maximal geometric genus, then by [D, Theorem (1.8)] one has also $h_{\Gamma}=h(3 ; d, s)$. In other words $V^{(1)}$ is a curve in $\mathbf{P}^{3}$ contained in a surface of degree $s$ and of "caractère maximum" in the sense of [E]. By [E, Proposition IV.2] (here we are forced to use the restriction $\epsilon \notin\{0,1, s-3\})$ it follows that $V^{(1)}$ is a.C.M. and so it is of maximal arithmetic genus with respect to the flag condition $(1,3 ; d, s)$. By [GP] we know that $V^{(1)}$ is linked in $\mathbf{P}^{3}$ to a plane curve of degree $s-1-\epsilon$ in a complete intersection of two surfaces of degrees $s$ and $m+1$, and such a linkage can be lifted in $\mathbf{P}^{n+2}$ because $V$ is a.C.M. The last assertion follows by [PS].

Remark 3.2. (i) If $V \subset \mathbf{P}^{r}$ is a variety with only canonical singularities then $p_{g}(V)=h^{0}\left(V, \omega_{V}\right)($ see $[\mathrm{R}]$ and $[\mathrm{K}])$.

(ii) Notice that, in the special case $n \geq 3, s \geq 3$ and $\epsilon \in\{0,1, s-3\}$, our classification of the varieties $V \in \mathcal{S}(n, n+2 ; d, s)$ of maximal geometric genus is not complete. In fact, in this case, we do not know whether a variety $V \in \mathcal{S}(n, n+2 ; d, s)$ of maximal geometric genus is a.C.M. and consequently linked to a degenerate variety in a complete intersection, as in Halphen's theorem for curves. However, by [D, Theorem (3.7) and proof] and taking into account [CCD1, Theorem (5.1)], we know that a surface $V \in \mathcal{S}(2,4 ; d, s)$, with $d>12 s^{2}$, nonsingular in codimension one and of maximal geometric genus $G(2,4 ; d, s)$, is a.C.M.. Hence in this case we do not need [E] in the proof of (iii) of the previous theorem, and so we can suppress the hypothesis $\epsilon \notin\{0,1, s-3\}$ and state Theorem (3.1) as follows.

Theorem 3.3. Let $V \subset \mathbf{P}^{4}$ be a surface of degree $d$, nonsingular in codimension one, such that $p_{g}(V)=h^{0}\left(V, \omega_{V}\right)$ (e.g. smooth or with only canonical singularities). Assume that $V$ is not contained in any hypersurface of degree $<s$, with $d>s^{2}-s$, $s \geq 2$. Put $d-1=m s+\epsilon, 0 \leq \epsilon<s$. Then one has $p_{g}(V) \leq G^{h}(2,4 ; d, s)$ and, when $d>12 s^{2}, p_{g}(V)=G^{h}(2,4 ; d, s)$ if and only if $V$ is linked to a space surface 
$V^{\prime}$ of degree $s-1-\epsilon$ in a complete intersection of two hypersurfaces of degrees $s$ and $m+1$.

As for the case of curves and surfaces, we believe that, in the case of $n \geq 3$, the restriction on $\epsilon$ can be suppressed. We hope to return to this question in a future paper.

As a consequence of the previous results and Lemma (2.1), we have the following:

Corollary 3.4. Let $V \subset \mathbf{P}^{n+2}$ be a variety of degree $d>\max \left\{4 s^{2},(n+s)^{2}\right\}$ and dimension $n \geq 2$, and let $H \subset \mathbf{P}^{n+1}$ be its generic hyperplane section. Assume that $p_{g}(V)=h^{0}\left(V, \omega_{V}\right)$ and $p_{g}(H)=h^{0}\left(H, \omega_{H}\right)$ (e.g. $V$ smooth or with only canonical singularities) and that $s=2$ or $n=2$ or $\epsilon \notin\{0,1, s-3\}$. Then $V \in \mathcal{S}(n, n+2 ; d, s)$ if and only if $H \in \mathcal{S}(n-1, n+1 ; d, s)$ and, in such a case, $p_{g}(V)=G(n, n+2 ; d, s)$ if and only if $p_{g}(H)=G(n-1, n+1 ; d, s)$.

Remark 3.5. (i) For any $d>s$, there are a.C.M. varieties $V \in \mathcal{S}(n, r ; d, s)$ such that $h^{0}\left(V, \omega_{V}\right)=G^{h}(n, r ; d, s)$. In fact, by [CCD1] we know that there are curves $C \in \mathcal{S}(1, r-n+1 ; d, s)$ of maximal arithmetic genus $p_{a}(C)=G^{h}(1, r-n+1 ; d, s)$. In particular, $h_{C^{(0)}}=h(r-n+1 ; d, s)$. Taking cones over these curves, we can construct the desired varieties $V$ (use Remark (2.3)). Observe that such varieties $V$ are ruled, and so $p_{g}(V)=0$.

(ii) Notice that

$$
G^{h}(n, n+2 ; d, s)=\frac{d^{n+1}}{(n+1) ! s^{n}}+\frac{d^{n}}{2 n ! s^{n-1}}(s-n-3)+O\left(d^{n-1}\right)
$$

with $\left|O\left(d^{n-1}\right)\right| \leq d^{n-1} s^{4-n}\left[3^{n+1} 4+2^{n-1} n(n+8)\right]$. Moreover, by [NV, Theorem 4], we have

$$
G^{h}(n, n+2 ; d, s)=\left(\begin{array}{c}
m+s-1 \\
n+2
\end{array}\right)+\left(\begin{array}{c}
m+\epsilon \\
n+1
\end{array}\right)-\left(\begin{array}{c}
m \\
n+2
\end{array}\right)-\left(\begin{array}{c}
s-4 \\
n+2
\end{array}\right) .
$$

\section{The CASE OF CODIMENSION THREE AND FURTHER RESULTS IN THE GENERAL CASE}

The next step is an analysis of the case $l=r-n=3$. As before, we start with some comments in a more general situation, that is, $l=r-n \geq 3$.

As we said in (1.5), when $l \geq 3$ the functions $h\left(r-n+1 ; s_{1}, \ldots, s_{l}\right)$ we introduced are LAF for $\mathcal{F}\left(1, r-n+1 ; s_{1}, \ldots, s_{l}\right)$ but not sharp. This leads us to expect that for $l \geq 3$ the upper bound $G^{h}\left(n, r ; s_{1}, \ldots, s_{l}\right)$ is not sharp. However, in the special case $r-n+1=4$ and $l=3$, one can refine such functions by using the functions $\tilde{h}\left(4 ; s_{1}, s_{2}, s_{3}\right)$ defined in (1.5), obtaining the sharp function for the set $\mathcal{F}\left(1,4 ; s_{1}, s_{2}, s_{3}\right)$ [CCD3]. By means of these new functions one can improve the upper bound $G^{h}\left(n, r ; s_{1}, \ldots, s_{r-n}\right)$ in the case $l=r-n$, in the following way (we assume throughout this section $\left.s_{1}>>\ldots>>s_{r-n}\right)$.

First one defines new functions $\tilde{h}\left(r-n+1 ; s_{1}, \ldots, s_{r-n}\right)$ by induction on $r-n$, putting for $r-n>3$

$$
\tilde{h}\left(r-n+1 ; s_{1}, \ldots, s_{r-n}\right)(i):=\sum_{j=0}^{i} \tilde{h}\left(r-n ; s_{2}, \ldots, s_{r-n}\right)(j) \quad \text { for } \quad 0 \leq i \leq a_{1}
$$


and

$$
\begin{aligned}
& \tilde{h}\left(r-n+1 ; s_{1}, \ldots, s_{r-n}\right)(i) \\
& \quad:=\min \left\{s_{1}, \tilde{h}\left(r-n+1 ; s_{1}, \ldots, s_{r-n}\right)\left(a_{1}\right)+\tilde{h}\left(r-n+1 ; s_{1}, \ldots, s_{r-n}\right)\left(i-a_{1}\right)-1\right\}
\end{aligned}
$$

for $i>a_{1}$.

By using a similar argument as in the proof of [CCD2, Proposition (1.1)] and taking into account [CCD3], one proves that

$$
h\left(r-n+1 ; s_{1}, \ldots, s_{r-n}\right) \leq \tilde{h}\left(r-n+1 ; s_{1}, \ldots, s_{r-n}\right) \leq s_{1}
$$

Next one defines

$$
G^{\tilde{h}}\left(n, r ; s_{1}, \ldots, s_{r-n}\right):=\sum_{i=1}^{\infty}\left(\begin{array}{c}
i-1 \\
n-1
\end{array}\right)\left(s_{1}-\tilde{h}\left(r-n+1 ; s_{1}, \ldots, s_{r-n}\right)(i)\right)
$$

and shows, in a similar way as for $G^{h}\left(n, r ; s_{1}, \ldots, s_{l}\right)$ (see Proposition A), that there exist rational numbers $\tilde{\eta}$ and $\widetilde{O}$ such that

$$
|\tilde{\eta}| \leq \frac{3}{4}, \quad|\widetilde{O}| \leq s_{1}^{n-1} \frac{s_{2}^{4-n}}{s_{3}^{2}}\left[4(r-n+1)^{n+1}+2^{n-1} n(n+8)\right]
$$

and that

$$
\begin{gathered}
G^{\tilde{h}}\left(n, r ; s_{1}, \ldots, s_{r-n}\right)=\frac{s_{1}{ }^{n+1}}{(n+1) ! s_{2}{ }^{n}} \\
+\frac{s_{1}{ }^{n}}{2 n ! s_{2}{ }^{n-1}}\left[\frac{s_{2}}{s_{3}}+\ldots+\frac{s_{r-n-1}}{s_{r-n}}+s_{r-n}-(r+1)+\tilde{\eta}\right]+\widetilde{O} .
\end{gathered}
$$

In particular, one gets, as in Proposition B, that

$$
\delta_{i} \leq G^{\tilde{h}}\left(n, r ; s_{1}, \ldots, s_{r-n}\right)-G^{h}\left(n, r ; s_{1}, \ldots, s_{i-1}, s_{i}+1\right)
$$

for all $i=2, \ldots, r-n$, from which, as in Section 2, one deduces the following:

Theorem 4.1. If $V \in \mathcal{S}\left(n, r ; s_{1}, \ldots, s_{r-n}\right)$ then $p_{g}(V) \leq G^{\tilde{h}}\left(n, r ; s_{1}, \ldots, s_{r-n}\right)$.

Taking into account (4.0.1) and the fact that for $i \in\left[a_{1}+1, a_{1}+a_{2}+a_{3}\right]$ one has $h\left(r-n+1 ; s_{1}, \ldots, s_{r-n}\right)(i)<\tilde{h}\left(r-n+1 ; s_{1}, \ldots, s_{r-n}\right)(i)$, one sees that Theorem (4.1) improves Theorem (2.5), (i), in the case $l=r-n \geq 3$.

As we are going to see, in codimension three and in some numerical range for the $s_{i}$ 's, the upper bound $G^{\tilde{h}}\left(n, n+3 ; s_{1}, s_{2}, s_{3}\right)$ is sharp. Next we will characterize the smooth complete intersections in $\mathbf{P}^{n+3}$ of type $(a, b, c)$, with $a>>b>>$, as the smooth varieties of maximal geometric genus with respect to the flag condition $(n, n+3 ; a b c, b c, c)$. In particular this applies to smooth surfaces in $\mathbf{P}^{5}$.

We keep the notation stated in (1.5), where we defined the functions $\tilde{h}\left(4 ; s_{1}, s_{2}, s_{3}\right)$. In particular we put $d=s_{1}, s=s_{2}$ and $t=s_{3}$.

Theorem 4.2. Fix integers $n \geq 1, d, s, t$ with $d>>s>>t, t \geq 2$. Assume that $s$ divides $d$. Then the upper bound $G^{\tilde{h}}(n, n+3 ; d, s, t)$ is sharp (and, when $1 \leq n \leq 3$, it is attained by smooth varieties). In other words:

$$
G(n, n+3 ; d, s, t)=G^{\tilde{h}}(n, n+3 ; d, s, t)=\max \left\{p_{g}(V): V \in \mathcal{S}(n, n+3 ; d, s, t)\right\} .
$$


In particular, in such a case, any variety $V \in \mathcal{S}(n, n+3 ; d, s, t)$ of maximal geometric genus must lie on a unique flag of type

$$
V=V_{d}^{n} \subset V_{s}^{n+1} \subset V_{t}^{n+2} \subset \mathbf{P}^{n+3} .
$$

For any such a variety $V$ one has $p_{g}(V)=h^{0}\left(V, \omega_{V}\right)$ and $h_{V^{(0)}}=\tilde{h}(4 ; d, s, t)$. Moreover the generic curve section $V_{s}^{(1)}$ of $V_{s}^{n+1}$ is a curve of maximal arithmetic genus with respect to the flag condition $(1,3 ; s, t)$.

Proof. Let $U^{\prime} \subset \mathbf{P}^{n+3}$ be a generic, degenerate subvariety of dimension $n+1$ and degree $t-1-\beta$, and consider $F, G$ generic hypersurfaces containing $U^{\prime}$ of degrees respectively $t$ and $\alpha+1$. Let $U$ be the variety linked to $U^{\prime}$ in the complete intersection $F \cap G$. Now let $H$ be a generic hypersurface of degree $m+1\left(=\frac{d}{s}\right)$ and put $M=F \cap G \cap H, V^{\prime}=U^{\prime} \cap H$ and $V=U \cap H$ (note that, by [PS], $V$ is smooth for $1 \leq n \leq 3$ ). By using a similar argument as in the proof of (ii) of Theorem (3.1) one sees that

$$
p_{g}(V) \geq h^{0}\left(\mathbf{P}^{n+3}, \mathcal{I}_{V^{\prime}}(\nu)\right)-h^{0}\left(\mathbf{P}^{n+3}, \mathcal{I}_{M}(\nu)\right)
$$

where $\nu=m+\alpha+t-n-2$. Let $\mathbf{P}^{3}$ be a generic linear 3-space of $\mathbf{P}^{n+3}$ and put $\Gamma^{\prime}=\mathbf{P}^{3} \cap V^{\prime}$ and $\bar{\Gamma}=\mathbf{P}^{3} \cap M$. As in Theorem (3.1) one has

$$
h^{0}\left(\mathbf{P}^{n+3}, \mathcal{I}_{V^{\prime}}(\nu)\right)-h^{0}\left(\mathbf{P}^{n+3}, \mathcal{I}_{M}(\nu)\right)=\sum_{i=0}^{\nu}\left(\begin{array}{c}
m+\alpha+t-3-i \\
n-1
\end{array}\right)\left(h_{\bar{\Gamma}}(i)-h_{\Gamma^{\prime}}(i)\right)
$$

and by direct computations one sees that, for all $i$,

$$
h_{\bar{\Gamma}}(i)-h_{\Gamma^{\prime}}(i)=d-\tilde{h}(4 ; d, s, t)(m+\alpha+t-2-i),
$$

from which we deduce

$$
\begin{gathered}
\sum_{i=0}^{\nu}\left(\begin{array}{c}
m+\alpha+t-3-i \\
n-1
\end{array}\right)\left(h_{\bar{\Gamma}}(i)-h_{\Gamma^{\prime}}(i)\right)=\sum_{j=n}^{m+\alpha+t-2}\left(\begin{array}{c}
j-1 \\
n-1
\end{array}\right)(d-\tilde{h}(4 ; d, s, t)(j)) \\
=G^{\tilde{h}}(n, n+3 ; d, s, t) .
\end{gathered}
$$

Then by (4.2.1) and Theorem (4.1) we get $p_{g}(V)=G^{\tilde{h}}(n, n+3 ; d, s, t)$, which proves that, at least in our range, the bound $G^{\tilde{h}}(n, n+3 ; d, s, t)$ is sharp.

Next consider any variety $V \in \mathcal{S}(n, n+3 ; d, s, t)$ of maximal geometric genus. The fact that it must lie on a flag follows by Theorem (2.5), (ii), taking into account (4.0.2). By using the existence of this flag one sees that ([CCD3, Proposition (15), (16) and Remark (17)])

$$
h_{V^{(0)}} \geq \tilde{h}(4 ; d, s, t) .
$$

Since $p_{g}(V)=G^{\tilde{h}}(n, n+3 ; d, s, t)$, then one has, by Lemma $(2.2)$, that $p_{g}(V)=$ $h^{0}\left(V, \omega_{V}\right)$ and

$$
h_{V^{(0)}}(i)=\tilde{h}(4 ; d, s, t)(i) \quad \text { for all } i \geq n .
$$

We have to show (4.2.3) for all $0 \leq i \leq n-1$. To do this notice that $\tilde{h}(4 ; d, s, t)(n)=$ $h_{V_{d}^{(0)}}(n)=$ (by Bezout's theorem) $=h_{V_{s}^{(1)}}(n) \geq($ by $[\mathrm{EH}]) \geq h_{V_{s}^{(1)}}(n-1)+$ $h_{V_{s}^{(0)}}(n) \geq($ by $[\mathrm{Hr} 1]) \geq h_{V_{s}^{(1)}}(n-1)+h(3 ; s, t)(n)=h_{V_{d}^{(0)}}(n-1)+h(3 ; s, t)(n) \geq$ 
(by $(4.2 .2)) \geq \tilde{h}(4 ; d, s, t)(n-1)+h(3 ; s, t)(n)=\tilde{h}(4 ; d, s, t)(n)$, from which we deduce that $h_{V_{d}^{(0)}}(n-1)=\tilde{h}(4 ; d, s, t)(n-1)$. Continuing in this fashion one proves (4.2.3) for all $0 \leq i \leq n-1$.

It remains to prove that the generic curve section $V_{s}^{(1)}$ of $V_{s}^{n+1}$ is a curve of maximal arithmetic genus with respect to the flag condition $(1,3 ; s, t)$. To this purpose, observe that, by [GLP], one has

$$
\begin{aligned}
p_{a}\left(V_{s}^{(1)}\right) & =n s+1-h_{V_{s}^{(1)}}(s)=n s+1-h_{V_{d}^{(0)}}(s) \\
& =n s+1-\tilde{h}(4 ; d, s, t)(s)=G^{h}(1,3 ; s, t) .
\end{aligned}
$$

Theorem 4.3. Let $V \subset \mathbf{P}^{n+3}$ be an irreducible, reduced, nondegenerate projective variety of dimension $n \geq 1$ and degree $d$, not contained in any hypersurface of degree $<t$ and in any $(n+1)$-dimensional variety of degree $\langle s$, with $d>>s>>t$, $t \geq 2$. Assume that $p_{g}(V)=h^{0}\left(V, \omega_{V}\right)$ (e.g. $V$ smooth or with only canonical singularities) and that $t$ divides $s$ and $s$ divides $d$. Then $V$ has maximal geometric genus with respect to the flag condition $(n, n+3 ; d, s, t)$ if and only if $V$ is a complete intersection of type $\left(t, \frac{s}{t}, \frac{d}{s}\right)$.

Proof. For a complete intersection $V$ of type $\left(t, \frac{s}{t}, \frac{d}{s}\right)$ one has $h_{V^{(0)}}=\tilde{h}(4 ; d, s, t)$ and so, by Remark (2.3) and the previous theorem, it follows that $V$ has maximal geometric genus.

Now assume that $p_{g}(V)=G(n, n+3 ; d, s, t)$. By the previous theorem we know that for such a variety one has $h_{V^{(0)}}=\tilde{h}(4 ; d, s, t)$, which is the Hilbert function of a complete intersection in $\mathbf{P}^{3}$ of type $\left(t, \frac{s}{t}, \frac{d}{s}\right)$. Combining this with the fact that $d>>s>t$ and that, again by the previous theorem, $V^{(0)}$ belongs to a flag, we have that $V^{(0)}$ is a complete intersection of type $\left(t, \frac{s}{t}, \frac{d}{s}\right)$ and so also $V$ is, by [Re, Teorema 2].

In the particular case of surfaces, i.e. $n=2$, we have the following:

Theorem 4.4. Let $V \subset \mathbf{P}^{5}$ be an irreducible, reduced, nondegenerate projective surface of degree $d$, not contained in any hypersurface of degree $<t$ and in any threefold of degree $<s$, with $d>>s>>t, t \geq 2$. Assume that $p_{g}(V)=h^{0}\left(V, \omega_{V}\right)$ (e.g. $V$ smooth or with only canonical singularities) and that $s$ divides $d$. Then $V$ has maximal geometric genus with respect to the flag condition $(2,5 ; d, s, t)$ if and only if $V$ is a complete intersection of a hypersurface $F$ of degree $d / s$ with a threefold $T$ of degree $s$, whose generic curve section $T^{(1)} \subset \mathbf{P}^{3}$ is a curve of maximal arithmetic genus with respect to the flag condition $(1,3 ; s, t)$. In particular, $V$ is a.C.M.

Proof. First assume that $V=F \cap T$, with $F$ and $T$ as in the statement. Then $V$ is a.C.M. and a direct computation shows that $h_{V^{(0)}}=\tilde{h}(4 ; d, s, t)$. By Remark $(2.3)$ and our hypotheses $p_{g}(V)=h^{0}\left(V, \omega_{V}\right)$, it follows that $p_{g}(V)=G^{\tilde{h}}(2,5 ; d, s, t)$, and so, by Theorem (4.2), $V$ is of maximal geometric genus.

Now assume that $V$ is of maximal geometric genus $p_{g}(V)=G(2,5 ; d, s, t)$. By Theorem (4.2), in order to show that $V$ is a complete intersection of type $V=F \cap T$, it suffices to prove that $V$ is a.C.M. To do this, by [D, Theorem (3.7) and proof], it is enough to show that the generic hyperplane section $C \subset \mathbf{P}^{4}$ of $V$ is linearly normal. 
Suppose not, and let $\xi$ be the linear system cut out on $C$ by the hyperplanes of $\mathbf{P}^{4}$. Let $\sigma$ be a linear system on $C$ such that $\xi \subset \sigma \subset\left|\mathcal{O}_{C}(1)\right|$, with $\operatorname{dim}(\sigma)=5$ $(=1+\operatorname{dim}(\xi))$. The linear system $\sigma$ determines in $\mathbf{P}^{5}$ a curve $\widetilde{C}$ of degree $d$ such that $C$ is a birational projection of $\widetilde{C}$ in $\mathbf{P}^{4}$. As well as the curve $C$, also $\widetilde{C}$ is not contained in any surface of $\mathbf{P}^{5}$ of degree $<s$. Hence, if we denote by $\widetilde{\Gamma}$ ( $\Gamma$ resp.) the generic hyperplane section of $\widetilde{C}(C$ resp. $)$, we have $h^{0}\left(C, \mathcal{O}_{C}(2)\right)-$ $h^{0}\left(C, \mathcal{O}_{C}(1)\right)=h^{0}\left(\widetilde{C}, \mathcal{O}_{\widetilde{C}}(2)\right)-h^{0}\left(\widetilde{C}, \mathcal{O}_{\widetilde{C}}(1)\right) \geq h_{\widetilde{\Gamma}}(2) \geq$ (by Proposition $\left.(2.4)\right)$ $\geq h(5 ; d, s)(2)=12>10 \geq \tilde{h}(4 ; d, s, t)(2)=$ (by Theorem $(4.2))=h_{\Gamma}(2)$. So we have

$$
h^{0}\left(C, \mathcal{O}_{C}(2)\right)-h^{0}\left(C, \mathcal{O}_{C}(1)\right)>h_{\Gamma}(2)
$$

On the other hand, since $p_{g}(V)=G^{\tilde{h}}(2,5 ; d, s, t)$, then

$$
p_{g}(V)=\sum_{i=1}^{\infty}(i-1)\left(d-h_{\Gamma}(i)\right) \text {. }
$$

This implies, again by $[\mathrm{D}$, Theorem $(3.7),(10)]$, that

$$
h^{0}\left(C, \mathcal{O}_{C}(2)\right)-h^{0}\left(C, \mathcal{O}_{C}(1)\right)=h_{\Gamma}(2),
$$

which is in contrast with (4.4.1).

As a consequence we have

Corollary 4.5. Fix integers $d>>s>t \geq 2$ and assume that $s$ divides $d$. Let $V \subset \mathbf{P}^{5}$ be an irreducible, reduced, nondegenerate projective surface of degree $d$, and let $H$ be its generic hyperplane section. Assume that $p_{g}(V)=h^{0}\left(V, \omega_{V}\right)$ (e.g. $V$ smooth or with only canonical singularities). Then $V$ satisfies a flag condition of type $(2,5 ; d, s, t)$ and has maximal geometric genus $G(2,5 ; d, s, t)$ if and only if $H$ satisfies a flag condition of type $(1,4 ; d, s, t)$ and has maximal arithmetic genus $G(1,4 ; d, s, t)$.

Proof. If $V \in \mathcal{S}(2,5 ; d, s, t)$ and $p_{g}(V)=G(2,5 ; d, s, t)$, then, by Theorem (4.2), $V$ lies on a flag and so $H$ satisfies a flag condition of type $(1,4 ; d, s, t)$. Moreover we have $h_{\Gamma}=\tilde{h}(4 ; d, s, t)$ and, by Theorem (4.4), we know that $H$ is a.C.M. By Remark (2.3) it follows that $p_{g}(V)=h^{0}\left(V, \omega_{V}\right)=G^{\tilde{h}}(1,4 ; d, s, t)$, which is equal to $G(1,4 ; d, s, t)$ by Theorem (4.2).

Conversely, if $H$ satisfies a flag condition of type $(1,4 ; d, s, t)$ and has maximal arithmetic genus $G(1,4 ; d, s, t)$ then, by [CCD3], $H$ is a.C.M. and $h_{\Gamma}=\tilde{h}(4 ; d, s, t)$. Hence $V$, which certainly belongs to $\mathcal{S}(2,5 ; d, s, t)$, is a.C.M. and so has maximal geometric genus by Remark (2.3) and Theorem (4.2).

Remark 4.6. (i) Consider the set $\mathcal{H}_{\mathcal{F}}\left(r ; s_{1}, \ldots, s_{l}\right):=\left\{h_{C^{(0)}}: C \in \mathcal{F}\left(1, r ; s_{1}, \ldots, s_{l}\right)\right\}$ of all Hilbert functions of the generic hyperplane sections of curves

$$
C \in \mathcal{F}\left(1, r ; s_{1}, \ldots, s_{l}\right) \text {. }
$$

Note that $\mathcal{H}_{\mathcal{F}}\left(r ; s_{1}, \ldots, s_{l}\right)$ is partially ordered by the ordering $h \leq h^{\prime} \Longleftrightarrow h(i) \leq$ $h^{\prime}(i)$ for all $i$. Consider the following property.

Property $(\alpha)$. For any $r, s_{1}, \ldots, s_{l}$ with $s_{1}>>\ldots>>s_{l}$ and such that $s_{i}$ divides $s_{i-1}$ for all $i=2, \ldots, l$, there exists the minimum function for the set $\mathcal{H}_{\mathcal{F}}\left(r ; s_{1}, \ldots, s_{l}\right)$.

Notice that Property $(\alpha)$ is certainly true when $l=2$ [CCD1] or $r=4$ [CCD3]. We do not know whether this is also true in the other cases. However, it may be 
interesting here to point out that Property $(\alpha)$ implies the generalization of the previous Theorem (4.3) to any codimension. More precisely, consider the following property.

Property $(\beta)$. Let $V \subset \mathbf{P}^{n+h}$ be a projective variety verifying a flag condition of type $\left(n, n+h ; s_{1}, \ldots, s_{h}\right)$, with $s_{1}>>\ldots>s_{h}$. Assume that $p_{g}(V)=h^{0}\left(V, \omega_{V}\right)$ and that $s_{i}$ divides $s_{i-1}$ for all $i=2, \ldots, h$. Then $V$ has maximal geometric genus $G\left(n, n+h ; s_{1}, \ldots, s_{h}\right)$ if and only if it is a complete intersection of type $\left(s_{l}, \frac{s_{l-1}}{s_{l}}, \ldots, \frac{s_{1}}{s_{2}}\right)$.

It is not hard to see that, if Property $(\alpha)$ holds, then the minimum function for the set $\mathcal{H}_{\mathcal{F}}\left(r ; s_{1}, \ldots, s_{l}\right)$ is the Hilbert function of a complete intersection of type $\left(s_{l}, \frac{s_{l-1}}{s_{l}}, \ldots, \frac{s_{1}}{s_{2}}\right)$ in $\mathbf{P}^{r-1}$. Then, by using a similar argument as in the proof of Theorem (4.3) (and taking into account Corollary (4.8) below), one can show that

$$
\text { Property }(\alpha) \Longrightarrow \text { Property }(\beta) \text {. }
$$

(ii) By using a similar argument as in Remark (3.5), (i), and the main result of [CCD3], one can see that, for all $d>>>>t, t \geq 2$, there exist varieties $V \in \mathcal{S}(n, n+3 ; d, s, t)$ such that $h^{0}\left(V, \omega_{V}\right)=G^{\tilde{h}}(n, n+3 ; d, s, t)$ and with geometric genus $p_{g}(V)=0$.

(iii) Notice that

$$
\begin{aligned}
G^{\tilde{h}}(n, n & +3 ; d, s, t) \\
= & \frac{d^{n+1}}{(n+1) ! s^{n}}+\frac{d^{n}}{2 n ! s^{n-1}}\left[\frac{s}{t}+t-(n+4)-\frac{(t-1-\beta)(1+\beta)(t-1)}{s t}\right] \\
& +O\left(d^{n-1}\right)
\end{aligned}
$$

with $\left|O\left(d^{n-1}\right)\right| \leq d^{n-1} \frac{s^{4-n}}{t^{2}}\left[4^{n+2}+2^{n-1} n(n+8)\right]$.

We conclude this section by showing that, in the general case $2 \leq l \leq r-n$ but only for some particular range for the $s_{i}$ 's, the varieties verifying flag conditions and of maximal genus $G\left(n, r ; s_{1}, \ldots, s_{l}\right)$ must lie on flags. We need the following lemma, but first we recall again that the number $\delta_{l}$, which appears in its statement, was defined in the statement of Proposition B (see $\S 2$ ).

Lemma 4.7. For any $r, n, s_{1}, \ldots, s_{l}$ with, as usual, $s_{1}>>\ldots>>s_{l}$, there exists a flag

$$
V=V_{s_{1}}^{n} \subset \ldots \subset V_{s_{l}}^{n+l-1} \subset \mathbf{P}^{r}
$$

of a.C.M. varieties such that $V_{s_{l}}^{(1)} \subset \mathbf{P}^{r-n-l+2}$ is a nondegenerate curve of maximal arithmetic genus.

For any such a flag there exist rational numbers $\bar{\eta}$ and $\bar{O}$ with $|\bar{\eta}| \leq \frac{3}{4}$ and $|\bar{O}| \leq s_{1}^{n-1} \frac{s_{2}^{4-n}}{s_{3}^{2}}\left[4(l+1)^{n+1}+2^{n-1} n(n+8)\right]$ such that

$$
\begin{aligned}
& h^{0}\left(V, \omega_{V}\right) \\
& =\frac{s_{1}{ }^{n+1}}{(n+1) ! s_{2}{ }^{n}}+\frac{s_{1}{ }^{n}}{2 n ! s_{2}{ }^{n-1}}\left[\frac{s_{2}}{s_{3}}+\ldots+\frac{s_{l}}{s_{l+1}}-l-\frac{2}{r-n+1-l}+\bar{\eta}-(n-1)\right] \\
& \quad+\bar{O} .
\end{aligned}
$$


In particular, if $p_{g}(V)=h^{0}\left(V, \omega_{V}\right)$, then

$$
G^{h}\left(n, r ; s_{1}, \ldots, s_{l}\right)-p_{g}(V) \leq \frac{3 s_{1}^{n}}{4 n ! s_{2}^{n-1}}+2 s_{1}^{n-1} \frac{s_{2}^{4-n}}{s_{3}^{2}}\left[4(l+1)^{n+1}+2^{n-1} n(n+8)\right]
$$

and so

$$
G^{h}\left(n, r ; s_{1}, \ldots, s_{l}\right)-p_{g}(V)<\delta_{l} .
$$

Proof. In order to show the existence of the flag, notice that, by using [CCD2, Lemma (2.6)] and the proof of [CCD2, Proposition (2.7)], one can construct a flag

$$
V_{s_{1}}^{1} \subset \ldots \subset V_{s_{l}}^{l} \subset \mathbf{P}^{r-n+1}
$$

of a.C.M. varieties such that, for the generic $(r-n+1-(l-1))$-dimensional subspace $L$ of $\mathbf{P}^{r-n+1}$, the curve $L \cap V_{s_{l}}^{l}$ is of maximal arithmetic genus in $L$. Taking the cone over this flag with vertex a generic $(n-1)$-dimensional subspace of $\mathbf{P}^{r}$, we obtain the desired flag.

Now we compute $h^{0}\left(V, \omega_{V}\right)$. To do this, first assume $n=1$ and $l=2$. In such a case we have $h^{0}\left(V, \omega_{V}\right)=p_{a}(V)$. By Remark (2.3) we know that $p_{a}\left(V_{s_{1}}^{1}\right)=$ $\sum_{i=1}^{\infty}\left(s_{1}-h_{V_{s_{1}}^{(0)}}(i)\right)$. By [CCD2, Proposition (2.4), (i)], this sum is equal to $\sum_{i=1}^{a_{1}+a_{2}+1}\left(s_{1}-h_{V_{s_{1}}^{(0)}}(i)\right)$. Put $I_{1}=\left[1, a_{2}+1\right], I_{2}=\left[a_{2}+2, a_{1}\right]$ and $I_{3}=\left[a_{1}+\right.$ $\left.1, a_{1}+a_{2}+1\right]$. One has $p_{a}\left(V_{s_{1}}^{1}\right)=\sum_{I_{1} \cup I_{2}}\left(s_{1}-h_{V_{s_{1}}^{(0)}}(i)\right)+\sum_{I_{3}}\left(s_{1}-h_{V_{s_{1}}^{(0)}}(i)\right)=$ $a_{1} s_{1}-\sum_{I_{1}} h_{V_{s_{1}}^{(0)}}(i)-\sum_{I_{2}} h_{V_{s_{1}}^{(0)}}(i)+\sum_{I_{3}}\left(s_{1}-h_{V_{s_{1}}^{(0)}}(i)\right)$. By Bezout's theorem we have $h_{V_{s_{1}}^{(0)}}(i)=h_{V_{s_{2}}^{(1)}}(i)$ for all $i \leq a_{1}$. Moreover, by [CCD2, Proposition (2.4), (ii)], and taking into account that $V_{s_{2}}^{(1)} \subset \mathbf{P}^{r-1}$ is a curve of maximal genus, we have $h_{V_{s_{2}}^{(1)}}(i)=1-G^{h}\left(1, r-1 ; s_{2}\right)+i s_{2}$ for all $i \geq a_{2}+2$ (here $G^{h}\left(1, r-1 ; s_{2}\right)$ denotes the upper bound for the genus of nondegenerate curves of degree $s_{2}$ in $\mathbf{P}^{r-1}$, found by Castelnuovo $[\mathrm{EH}])$. It follows that

$p_{a}\left(V_{s_{1}}^{1}\right)=a_{1} s_{1}-\sum_{I_{1}} h_{V_{s_{1}}^{(0)}}(i)-\sum_{I_{2}}\left(1-G^{h}\left(1, r-1 ; s_{2}\right)+i s_{2}\right)+\sum_{I_{3}}\left(s_{1}-h_{V_{s_{1}}^{(0)}}(i)\right)$.

And so, in order to evaluate $p_{a}\left(V_{s_{1}}^{1}\right)$, one can use the same calculations as in [CCD2, Proposition (1.1)] and gets rational numbers $|\eta| \leq \frac{3}{4}$ and $|\rho| \leq \frac{17 s_{2}^{3}}{(r-2)^{2}}$ such that

$$
p_{a}\left(V_{s_{1}}^{1}\right)=\frac{s_{1}^{2}}{2 s_{2}}+\frac{s_{1}}{2}\left[\frac{s_{2}}{r-2}-2-\frac{2}{r-2}+\eta\right]+\rho+1 .
$$

Now, in the case $n=1$ and $l \geq 3$, by using induction on $l$ as in [CCD2, Proposition (1.1)], one can show that

$$
p_{a}\left(V_{s_{1}}^{1}\right)=\frac{s_{1}^{2}}{2 s_{2}}+\frac{s_{1}}{2}\left[\frac{s_{2}}{s_{3}}+\ldots+\frac{s_{l}}{s_{l+1}}-l-\frac{2}{r-l}+\eta\right]+\rho+1,
$$

where $\eta$ and $\rho$ are suitable rational numbers such that $|\eta| \leq \frac{3}{4}$ and $|\rho| \leq$ $\left(l^{2}+2 l+9\right) s_{2}^{3} / s_{3}^{2}$. Finally the case $n \geq 2$ follows exactly as in the proof of our Proposition A (see the Appendix), taking into account that, by Remark (2.3), one has

$$
h^{0}\left(V_{s_{1}}^{n}, \omega_{V_{s_{1}}^{n}}\right)=\sum_{i=1}^{\infty}\left(\begin{array}{c}
i-1 \\
n-1
\end{array}\right)\left(d-h_{\Gamma}(i)\right)
$$


where $\Gamma$ is the intersection of $V_{s_{1}}^{n}$ with the generic $(r-n)$-dimensional subspace of $\mathbf{P}^{r}$.

Now, by using our formulas for $G^{h}\left(n, r ; s_{1}, \ldots, s_{l}\right), \delta_{l}$ and $h^{0}\left(V, \omega_{V}\right)$, and the numerical assumptions on the $s_{i}$ 's, one easily obtains the inequalities claimed in the last assertion.

Corollary 4.8. As usual, assume $s_{1}>>\ldots>>s_{l}$. Moreover suppose that at least one of the following conditions is satisfied.

(a) $s_{i}$ divides $s_{i-1}$ for all $i=2, \ldots, l$ and $s_{l} \geq(n+l-1)(r-n-l+1)+2$;

(b) $l=r-n$ and $s_{i}$ divides $s_{i-1}$ for all $i=2, \ldots, l-1$;

(c) $r \geq 2(n+l-2)+3, s_{l} \geq(n+l-1)(r-n-l+1)+2,0<b_{l}<r-n-l+1$, $b_{l-1} \geq a_{l}\left(r-n-l+1-b_{l}\right)$ and $s_{i}$ divides $s_{i-1}$ for all $i=2, \ldots, l-1$.

Then there exist varieties $V \in \mathcal{S}\left(n, r ; s_{1}, \ldots, s_{l}\right)$ such that

$$
G^{h}\left(n, r ; s_{1}, \ldots, s_{l}\right)-p_{g}(V) \leq \frac{3 s_{1}^{n}}{4 n ! s_{2}^{n-1}}+2 s_{1}^{n-1} \frac{s_{2}^{4-n}}{s_{3}^{2}}\left[4(l+1)^{n+1}+2^{n-1} n(n+8)\right] .
$$

It follows that $G^{h}\left(n, r ; s_{1}, \ldots, s_{l}\right)-G\left(n, r ; s_{1}, \ldots, s_{l}\right)<\delta_{l}$ and therefore the varieties $U$ verifying a flag condition of type $\left(n, r ; s_{1}, \ldots, s_{l}\right)$ and having maximal geometric genus $G\left(n, r ; s_{1}, \ldots, s_{l}\right)$ must lie on a flag of type $U=U_{s_{1}}^{n} \subset \ldots \subset U_{s_{l}}^{n+l-1} \subset \mathbf{P}^{r}$. In other words, in the previous ranges, one has:

$$
G\left(n, r ; s_{1}, \ldots, s_{l}\right)=\max \left\{p_{g}(U): U \in \mathcal{F}\left(n, r ; s_{1}, \ldots, s_{l}\right)\right\} .
$$

Proof. By Lemma (4.7), by Lemma (2.1), (1), and by Theorem (2.5), (ii), in order to show our claim, it suffices to construct a flag such as (4.7.1), with $p_{g}(V)=$ $h^{0}\left(V, \omega_{V}\right)$.

Assume (a) holds. Fix in $\mathbf{P}^{r}$ a Castelnuovo variety $V_{s_{l}}^{n+l-1}$ [Hr2]. One has that $V_{s_{l}}^{(1)} \subset \mathbf{P}^{r-n-l+2}$ is a smooth curve of maximal genus. And it is known that $V_{s_{l}}^{n+l-1}$ is a.C.M. and that $p_{g}\left(V_{s_{l}}^{n+l-1}\right)=h^{0}\left(V_{s_{l}}^{n+l-1}, \omega_{V_{s_{l}}^{n+l-1}}\right)$. If we take the intersection of $V_{s_{l}}^{n+l-1}$ with a generic hypersurface of degree $\frac{s_{l-1}}{s_{l}}$, then we get a variety $V_{s_{l-1}}^{n+l-2} \subset V_{s_{l}}^{n+l-1}$ which again satisfies these properties. Next we take the intersection of $V_{s_{l-1}}^{n+l-2}$ with a generic hypersurface of degree $\frac{s_{l-2}}{s_{l-1}}$ and get a variety $V_{s_{l-2}}^{n+l-3} \subset V_{s_{l-1}}^{n+l-2} \subset V_{s_{l}}^{n+l-1}$ with the same properties as before. Continuing in this manner we obtain the flag.

In case (b), for any $s_{r-n-1}>>s_{r-n} \geq 2$, by Theorem (3.1), (ii) and its proof, we can start by a flag $V_{s_{r-n-1}}^{r-2} \subset V_{s_{r-n}}^{r-1}$, where $V_{s_{r-n-1}}^{r-2}$ is a variety linked to a generic complete intersection of type $\left(1, s_{r-n}-1-b_{r-n-1}\right)$, by a complete intersection of two generic hypersurfaces $F\left(=V_{s_{r-n}}^{r-1}\right)$ and $G$, of degree respectively $s_{r-n}$ and $a_{r-n-1}+1$. Note that $V_{s_{r-n}}^{(1)}$ is a plane curve and so of maximal genus. Moreover we know that $V_{s_{r-n-1}}^{r-2}$ is a.C.M. and that $p_{g}\left(V_{s_{r-n-1}}^{r-2}\right)=h^{0}\left(V_{s_{r-n-1}}^{r-2}, \omega_{V_{s_{r-n-1}}^{r-2}}\right)$. As before, in order to construct the flag, we take the intersection of $V_{s_{r-n-1}}^{r-2}$ with a generic hypersurface of degree $\frac{s_{r-n-2}}{s_{r-n-1}}$ and get a variety $V_{s_{r-n-2}}^{r-3} \subset V_{s_{r-n-1}}^{r-2} \subset V_{s_{r-n}}^{r-1}$. Continuing in the same manner we construct a flag of a.C.M. varieties $V_{s_{i-n+1}}^{i}$, $i=n, \ldots, r-1$, and, by using a similar argument as in Theorem (4.2), (4.2.1), one sees that $p_{g}\left(V_{s_{i-n+1}}^{i}\right)=h^{0}\left(V_{s_{i-n+1}}^{i}, \omega_{V_{s_{i-n+1}}^{i}}\right)$ for all $i=n, \ldots, r-1$.

Finally, in case (c), by [D, Example (4.2)], we know that, when $s_{l-1}>>s_{l}$, there exists a flag $V_{s_{l-1}}^{n+l-2} \subset V_{s_{l}}^{n+l-1}$ of smooth and a.C.M. varieties, with $V_{s_{l}}^{n+l-1}$ 
of Castelnuovo's. As before, one extends the previous flag by taking intersections with generic hypersurfaces of appropriate degree.

Corollary 4.9. With the same assumptions as in Corollary (4.8), one has

$$
G\left(n, r ; s_{1}, \ldots, s_{i}+1\right)<G\left(n, r ; s_{1}, \ldots, s_{l}\right) \quad \text { for all } i=2, \ldots, l .
$$

Proof. Combining Theorem (2.5) and Proposition B with the previous corollary, we have

$$
\begin{gathered}
G\left(n, r ; s_{1}, \ldots, s_{i}+1\right) \leq G^{h}\left(n, r ; s_{1}, \ldots, s_{i}+1\right) \leq G^{h}\left(n, r ; s_{1}, \ldots, s_{l}\right)-\delta_{i} \\
\leq G^{h}\left(n, r ; s_{1}, \ldots, s_{l}\right)-\delta_{l}<G\left(n, r ; s_{1}, \ldots, s_{l}\right) .
\end{gathered}
$$

Remark 4.10. (i) Note that property (4.9.1) implies (4.8.1).

(ii) With the same assumptions as in Corollary (4.8), we deduce that, for a suitable rational number $\eta^{*}$, with $\left|\eta^{*}\right| \leq 3 / 2$, one has

$$
\begin{gathered}
G\left(n, r ; s_{1}, \ldots, s_{l}\right)=\frac{s_{1}{ }^{n+1}}{(n+1) ! s_{2}{ }^{n}} \\
+\frac{s_{1}{ }^{n}}{2 n ! s_{2}{ }^{n-1}}\left[\frac{s_{2}}{s_{3}}+\ldots+\frac{s_{l}}{s_{l+1}}-l-\frac{2}{r-n+1-l}+\eta^{*}-(n-1)\right]+O\left(s_{1}^{n-1}\right) .
\end{gathered}
$$

5. Some bounds for Castelnuovo's Regularity OF VARIETIES VERIFYING FLAG CONDITIONS

In this section we establish some bounds for Castelnuovo's regularity of the varieties $V \in \mathcal{S}\left(n, r ; s_{1}, s_{2}, \ldots, s_{l}\right)$ under the assumption that $V$ is a.C.M. (see Theorem (5.2) below) or that $l=2$ (Theorem (5.3)), extending [CCD2, Proposition (2.4)] to higher dimensional varieties. First we show the following easy consequence of [CCD2, Proposition (2.4)] and our Lemma (2.1).

Lemma 5.1. Assume $V \in \mathcal{S}\left(n, r ; s_{1}, \ldots, s_{l}\right)$, with $s_{1}>>\ldots>>s_{l}$. As in (1.3), define the numbers $a_{1}, \ldots, a_{l}$ by dividing $s_{i}-1=a_{i} s_{i+1}+b_{i}, 0 \leq b_{i}<s_{i+1}$, for all $i=1, \ldots, l$, where $s_{l+1}:=r-n-l+1$. Then one has:

(1) $H^{1}\left(\mathbf{P}^{r}, \mathcal{I}_{V^{(0)}}(i)\right)=0$ for all $i \geq a_{1}+\ldots+a_{l}+2$;

(2) $H^{j}\left(\mathbf{P}^{r}, \mathcal{O}_{V^{(j)}}(i)\right)=0$ for all $j=1, \ldots, n$ and $i \geq a_{1}+\ldots+a_{l}+2-j$.

Proof. In order to prove (1), consider $V^{(1)}$. By Lemma (2.1), (3), we know that $V^{(1)} \in \bigcup_{i=2}^{l} \widetilde{\mathcal{F}}\left(1, r-n+1 ; s_{1}, s_{2}, \ldots, s_{i}+1\right) \cup \mathcal{F}\left(1, r-n+1 ; s_{1}, s_{2}, \ldots, s_{l}\right)$. If $V^{(1)} \in \mathcal{F}\left(1, r-n+1 ; s_{1}, s_{2}, \ldots, s_{l}\right)$ then, by [CCD2, Proposition (2.4)], we have (1). Otherwise, if $V^{(1)} \in \widetilde{\mathcal{F}}\left(1, r-n+1 ; s_{1}, s_{2}, \ldots, s_{i}+1\right)$ for some $i=2, \ldots, l$, then, again by [CCD2, Proposition (2.4)], we have that $H^{1}\left(\mathbf{P}^{r}, \mathcal{I}_{V^{(0)}}(i)\right)=0$ for all $i \geq \bar{a}_{1}+\ldots+\bar{a}_{i}+2$, where $\bar{a}_{1}, \ldots, \bar{a}_{i}$ are the corresponding numbers $a_{1}, \ldots, a_{l}$ with respect to the sequence $s_{1}, \ldots, s_{i}+1$. Now property (1) follows by observing that, by our numerical assumptions, one has $\bar{a}_{1}+\ldots+\bar{a}_{i}+2 \leq a_{1}+\ldots+a_{l}+2$.

To prove (2), first note that the case $j=1$ is an immediate consequence of (1). Hence assume $j \geq 2$ and notice that our vanishing follows by induction on $j$, by using the natural exact sequence:

$$
H^{j-1}\left(\mathbf{P}^{r}, \mathcal{O}_{V^{(j-1)}}(i)\right) \rightarrow H^{j}\left(\mathbf{P}^{r}, \mathcal{O}_{V^{(j)}}(i-1)\right) \rightarrow H^{j}\left(\mathbf{P}^{r}, \mathcal{O}_{V^{(j)}}(i)\right) \rightarrow 0 .
$$


As an immediate consequence we have the following theorem which, in the case of codimension two and for $s_{1}>>s_{2} \geq 3$, improves [NV, Corollary 15].

Theorem 5.2. Let $V \subset \mathbf{P}^{r}$ be an a.C.M. variety with $V \in \mathcal{S}\left(n, r ; s_{1}, \ldots, s_{l}\right), s_{1}>>$ $\ldots>>s_{l}$. Define the numbers $a_{1}, \ldots, a_{l}$ by dividing $s_{i}-1=a_{i} s_{i+1}+b_{i}, 0 \leq b_{i}<s_{i+1}$, for all $i=1, \ldots, l$, where $s_{l+1}:=r-n-l+1$. Then $V$ is $\left(a_{1}+\ldots+a_{l}+3\right)$-regular (in the sense of Castelnuovo-Mumford). In particular the defining ideal $I(V)$ of $V$ is generated by forms of degree $\leq a_{1}+\ldots+a_{l}+3$.

Now we show another regularity result, whose proof only works for $l=2$. As in Section 3, put $d=s_{1}, s=s_{2}, m=a_{1}, w=a_{2}$ and assume $V \in \mathcal{S}(n, r ; d, s)$. Notice that, by Lemma (2.1), (4), we know that $V^{(i)} \in \mathcal{S}(i, r-n+i ; d, s)$ for all $i=1, \ldots, n$. Put $\mu\left(V^{(0)}\right):=m+w+3$ and, for $i=1, \ldots, n$,

$$
\begin{aligned}
\mu\left(V^{(i)}\right):= & \mu\left(V^{(i-1)}\right) \\
+ & {\left[\sum_{j=0}^{i-1}(-1)^{i+1+j}\left(\begin{array}{c}
\tau+j-1 \\
j
\end{array}\right)\right.} \\
& \left.\quad \times\left(G^{h}(i-j, r-n+i-j ; d, s)-p_{a}\left(V^{(i-j)}\right)\right)\right]_{\tau=\mu\left(V^{(i-1)}\right)-1}
\end{aligned}
$$

Recall that, when $n=1, p_{a}(V) \leq G^{h}(1, r ; d, s)$ [CCD1], and that for any $d>>s$ there are varieties $V \in \mathcal{S}(n, r ; d, s)$ such that $p_{a}\left(V^{(n-j)}\right)=G^{h}(n-j, r-j ; d, s)$ for all $j=0, \ldots, n-1$ (Remark (3.5), (i)). Now we are going to show the following theorem, which should be compared with [D, Theorem (2.1)].

Theorem 5.3. Let $V \subset \mathbf{P}^{r}$ be a projective variety and assume that $V \in \mathcal{S}(n, r ; d, s)$ and $d>>$ s. Put $\mu:=\mu(V)$ and $\mu^{\prime}:=\mu\left(V^{(n-1)}\right)$. Then the following properties hold.

(1) $V$ is $\mu$-regular.

(2) $\mu \geq \mu^{\prime} \geq m+w+3$.

(3) For all $\tau \geq \mu^{\prime}-1$ one has

$$
\sum_{j=0}^{n-1}(-1)^{n+1+j}\left(\begin{array}{c}
\tau+j-1 \\
j
\end{array}\right)\left[G^{h}(n-j, r-j ; d, s)-p_{a}\left(V^{(n-j)}\right)\right] \geq 0 .
$$

Proof. We will use induction on the dimension $n$. Notice that the case $n=1$ follows by [CCD2, Proposition (2.4)]. Hence we only have to analyse the case $n \geq 2$. Recall that, in order to show property (1), we have to prove

$$
H^{i}\left(\mathbf{P}^{r}, \mathcal{I}_{V}(\mu-i)\right)=0 \text { for all } i=1, \ldots, r .
$$

First we show (5.3.1) for $i=1$. Since $V^{(n-1)} \in \mathcal{S}(n-1, r-1 ; d, s)$, then, by induction, the homogeneous ideal of $V^{(n-1)}$ is generated in degree $t \leq \mu^{\prime}$. In a similar way as in [CCD2, Proposition (2.4)], it follows that for any $t \geq \mu^{\prime}-1$ one has $h^{1}\left(\mathbf{P}^{r}, \mathcal{I}_{V}(t+1)\right) \leq \max \left\{0, h^{1}\left(\mathbf{P}^{r}, \mathcal{I}_{V}(t)\right)-1\right\}$, from which we obtain $h^{1}\left(\mathbf{P}^{r}, \mathcal{I}_{V}(t)\right)=0$, for all $t \geq \mu^{\prime}-1+h^{1}\left(\mathbf{P}^{r}, \mathcal{I}_{V}\left(\mu^{\prime}-1\right)\right)$. So, in order to prove (5.3.1) for $i=1$, we only need to show that

$$
\begin{aligned}
& h^{1}\left(\mathbf{P}^{r}, \mathcal{I}_{V}\left(\mu^{\prime}-1\right)\right) \\
& \quad \leq \sum_{j=0}^{n-1}(-1)^{n+1+j}\left(\begin{array}{c}
\mu^{\prime}+j-2 \\
j
\end{array}\right)\left[G^{h}(n-j, r-j ; d, s)-p_{a}\left(V^{(n-j)}\right)\right] .
\end{aligned}
$$


For this purpose notice that, by using Lemma (5.1) and the fact that $\mu^{\prime} \geq m+w+3$, we have

$$
h^{1}\left(\mathbf{P}^{r}, \mathcal{I}_{V}\left(\mu^{\prime}-1\right)\right)=h^{0}\left(V, \mathcal{O}_{V}\left(\mu^{\prime}-1\right)\right)-h_{V}\left(\mu^{\prime}-1\right)=p_{V}\left(\mu^{\prime}-1\right)-h_{V}\left(\mu^{\prime}-1\right)
$$

by [D, Lemma (2.4)]

$$
\leq p_{V}\left(\mu^{\prime}-1\right)-\sum_{j=0}^{\mu^{\prime}-1}\left(\begin{array}{c}
\mu^{\prime}+n-j-2 \\
n-1
\end{array}\right) h_{V^{(0)}}(j)
$$

by Proposition (2.4)

$$
\leq p_{V}\left(\mu^{\prime}-1\right)-\sum_{j=0}^{\mu^{\prime}-1}\left(\begin{array}{c}
\mu^{\prime}+n-j-2 \\
n-1
\end{array}\right) h(r-n+1 ; d, s)(j)
$$

by [D, Corollary (2.9)]

$$
=\sum_{j=0}^{n-1}(-1)^{n+1+j}\left(\begin{array}{c}
\mu^{\prime}+j-2 \\
j
\end{array}\right)\left[G^{h}(n-j, r-j ; d, s)-p_{a}\left(V^{(n-j)}\right)\right] .
$$

This shows (5.3.2) and so (5.3.1) for $i=1$. Notice that property (2) follows by (5.3.2). In a similar way one proves property (3) for $\tau \geq \mu^{\prime}-1$.

Finally observe that, in the case $i \geq 2$, by using the natural exact sequences

$$
\begin{aligned}
H^{i-1}\left(V, \mathcal{O}_{V}(j-1)\right) & \rightarrow H^{i}\left(\mathbf{P}^{r}, \mathcal{I}_{V}(j-1)\right) \\
H^{i-2}\left(V^{(n-1)}, \mathcal{O}_{V^{(n-1)}}(j)\right) & \rightarrow H^{i-1}\left(\mathbf{P}^{r}, \mathcal{O}_{\mathbf{P}^{r}}(j-1)\right), \\
& \rightarrow H^{i-1}\left(V^{(n-1)}, \mathcal{O}_{V^{(n-1)}}(j)\right),
\end{aligned}
$$

one deduces the vanishing claimed in (5.3.1) as a consequence of the $\mu^{\prime}$-regularity of $V^{(n-1)}$, of Lemma (5.1) and of property (2).

Remark 5.4. By taking into account the generalized Laudal's lemma [St], and the proof of previous theorems, one has that, in the case of codimension two, i.e. $r-n=$ 2 , all the results in this section hold for $d>s^{2}+s$.

Now fix a surface $V$ of degree $d$ in $\mathbf{P}^{4}$, not lying on an hypersurface of degree $<s$, with $d>s^{2}+s$, and put $\gamma=G^{h}(1,3 ; d, s)-p_{a}\left(V^{(1)}\right)$. By the previous Theorem (5.3), (3), we have $p_{a}(V) \geq G^{h}(2,4 ; d, s)-\gamma((d-1) / s+s+1+\gamma)$. By direct computations one sees that

$$
\begin{aligned}
G^{h}(2,4 ; d, s) \geq & d^{3} / 6 s^{2}+d^{2}(s-5) / 4 s+d\left(2 s^{3}-21 s^{2}+29 s+12\right) / 12 s \\
& -\left(2 s^{5}+9 s^{4}-5 s^{3}+10 s^{2}-4\right) / 12 s^{2},
\end{aligned}
$$

and so we get the following lower bound for the Euler characteristic $\chi\left(\mathcal{O}_{V}\right)$ of $V$ :

$$
\begin{aligned}
\chi\left(\mathcal{O}_{V}\right) \geq & d^{3} / 6 s^{2}+d^{2}(s-5) / 4 s+d\left(2 s^{3}-21 s^{2}+29 s+12\right) / 12 s \\
& -\left(2 s^{5}+9 s^{4}-5 s^{3}-2 s^{2}-4\right) / 12 s^{2}-\gamma((d-1) / s+s+1+\gamma)
\end{aligned}
$$


(see also $[\mathrm{J}]$ for the case $s=2$ ). Under the same hypotheses, but with $d>s^{2}-s$, another type of lower bound is the following [D, Theorem (2.1) and proof]:

$$
\begin{aligned}
\chi\left(\mathcal{O}_{V}\right) \geq d^{3} / 6 s^{2}+d^{2}(s-5) / 4 s+ & d\left(2 s^{3}-21 s^{2}+29 s+12\right) / 12 s \\
& -\left(2 s^{5}+9 s^{4}-5 s^{3}-2 s^{2}-4\right) / 12 s^{2}-t \gamma,
\end{aligned}
$$

where $t$ is any integer $\geq m+s-1$ such that $p_{V}(t) \geq h_{V}(t)$ (and so, by [GLP], we may take $t=d-3)$.

Both inequalities (5.4.1) and (5.4.2) should be compared with the lower bound [BF, (1.1), (e)], stated in a different way and for the case when $V$ is smooth and $s$ is the minimal degree of a hypersurface containing it. For instance, with these hypotheses, it is know (by $[\mathrm{EP}]$ ) that $\gamma \leq d(s-1)^{2} / 2 s$. Hence we may replace $\gamma$ with $d(s-1)^{2} / 2 s$ in (5.4.2) (and $t$ with $d-3$ ), and get

$$
\begin{aligned}
\chi\left(\mathcal{O}_{V}\right) \geq d^{3} / 6 s^{2}-d^{2}\left(2 s^{2}-5 s+7\right) / 4 s+ & d\left(2 s^{3}-3 s^{2}-7 s+30\right) / 12 s \\
- & \left(2 s^{5}+9 s^{4}-5 s^{3}-2 s^{2}-4\right) / 12 s^{2},
\end{aligned}
$$

which is, for $d>s^{2}-s$ and $s \geq 5$, a lower bound better than the corresponding bound of [BF, (1.1), (e)]. Moreover, for $d>>s \geq 13$ and small values of $\gamma$, also the inequality (5.4.1) improves [BF, (1.1), (e)].

\section{APPENDIX}

The purpose of this appendix is to prove Propositions A and B claimed in Section 2. We keep all the notation and conventions stated in (1.3), (1.4) and (1.5), and refer to all the numerical properties of the functions $h\left(r-n+1 ; s_{1}, \ldots, s_{l}\right)$ from [CCD2, Proposition (1.1)]. In particular, recall that given the integers $s_{1}, \ldots, s_{l}$, we define the numbers $a_{i}$ and $b_{i}$ by dividing $s_{i}-1=a_{i} s_{i+1}+b_{i}, 0 \leq b_{i}<s_{i+1}$, for all $i=1, \ldots, l$, where $s_{l+1}:=r-n-l+1$.

Proof of Proposition A. For the case $n=1$ we refer to [CCD2, Proposition (1.1)]. Hence we assume $n \geq 2$.

In order to simplify our notation, first put $h:=h\left(r-n+1 ; s_{1}, \ldots, s_{l}\right)$. Next consider the following intervals of integer numbers:

$$
\begin{gathered}
I_{1}:=\left[1, a_{2}+\ldots+a_{l}+1\right], \\
I_{2}:=\left[a_{2}+\ldots+a_{l}+2, a_{1}\right], \\
I_{3}:=\left[a_{1}+1, a_{1}+\ldots+a_{l}+1\right] .
\end{gathered}
$$

By [CCD2, loc. cit.], we know that

$$
G^{h}\left(n, r ; s_{1}, \ldots, s_{l}\right)=\sum_{I_{1} \cup I_{2}}\left(\begin{array}{c}
i-1 \\
n-1
\end{array}\right)\left(s_{1}-h(i)\right)+\sum_{I_{3}}\left(\begin{array}{c}
i-1 \\
n-1
\end{array}\right)\left(s_{1}-h(i)\right) .
$$

We first compute $\sum_{I_{1} \cup I_{2}}\left(\begin{array}{c}i-1 \\ n-1\end{array}\right)\left(s_{1}-h(i)\right)$. For this purpose observe that

$$
\sum_{I_{1} \cup I_{2}}\left(\begin{array}{c}
i-1 \\
n-1
\end{array}\right)\left(s_{1}-h(i)\right)=\sum_{i=1}^{a_{1}}\left(\begin{array}{c}
i-1 \\
n-1
\end{array}\right) s_{1}-\sum_{I_{1}}\left(\begin{array}{c}
i-1 \\
n-1
\end{array}\right) h(i)-\sum_{I_{2}}\left(\begin{array}{c}
i-1 \\
n-1
\end{array}\right) h(i) .
$$


We analyse these three terms. First we have, taking into account that $a_{1}=\frac{s_{1}-b_{1}-1}{s_{2}}$, $0 \leq b_{1}<s_{2}$,

$$
\sum_{i=1}^{a_{1}}\left(\begin{array}{c}
i-1 \\
n-1
\end{array}\right) s_{1}=\left(\begin{array}{c}
a_{1} \\
n
\end{array}\right) s_{1}=\frac{s_{1}}{n !}\left[\frac{s_{1}^{n}}{s_{2}^{n}}-n \frac{s_{1}^{n-1}}{s_{2}^{n}}\left(b_{1}+1\right)-\left(\begin{array}{c}
n \\
2
\end{array}\right) \frac{s_{1}^{n-1}}{s_{2}^{n-1}}\right]+O_{1}+O_{2}
$$

where

$$
\begin{gathered}
O_{1}:=s_{1}\left[\left(\begin{array}{c}
a_{1} \\
n
\end{array}\right)-\frac{1}{n !}\left(a_{1}^{n}-\left(\begin{array}{c}
n \\
2
\end{array}\right) a_{1}^{n-1}\right)\right], \\
O_{2}:=\frac{s_{1}}{n !}\left[\sum_{i=2}^{n}\left(\begin{array}{c}
n \\
i
\end{array}\right)\left(\frac{s_{1}}{s_{2}}\right)^{n-i}\left(\frac{-b_{1}-1}{s_{2}}\right)^{i}-\left(\begin{array}{c}
n \\
2
\end{array}\right) \sum_{i=1}^{n-1}\left(\begin{array}{c}
n-1 \\
i
\end{array}\right)\left(\frac{s_{1}}{s_{2}}\right)^{n-1-i}\left(\frac{-b_{1}-1}{s_{2}}\right)^{i}\right],
\end{gathered}
$$

and, by elementary calculations which we omit,

$$
\left|O_{1}\right| \leq(n-2) \frac{s_{1}^{n-1}}{s_{2}^{n-2}}, \quad\left|O_{2}\right| \leq 4 \frac{s_{1}^{n-1}}{s_{2}^{n-2}} .
$$

Now we estimate

$$
O_{3}:=-\sum_{I_{1}}\left(\begin{array}{c}
i-1 \\
n-1
\end{array}\right) h(i)
$$

We have

$$
\sum_{I_{1}}\left(\begin{array}{c}
i-1 \\
n-1
\end{array}\right) h(i)=\sum_{i=1}^{a_{2}+\ldots+a_{l}}\left(\begin{array}{c}
i-1 \\
n-1
\end{array}\right)\left[\sum_{j=0}^{i} h\left(r-n ; s_{2}, \ldots, s_{l}\right)(j)\right] \leq
$$

(by [CCD2, loc. cit., (ii)]

$$
\begin{aligned}
& \leq \sum_{i=1}^{a_{2}+\ldots+a_{l}}\left(\begin{array}{c}
i-1 \\
n-1
\end{array}\right)\left[\sum_{j=0}^{i} s_{2}\right]=\sum_{i=1}^{a_{2}+\ldots+a_{l}}\left(\begin{array}{c}
i-1 \\
n-1
\end{array}\right) s_{2}+\sum_{i=1}^{a_{2}+\ldots+a_{l}}\left(\begin{array}{c}
i-1 \\
n-1
\end{array}\right) i s_{2} \\
& =s_{2}\left(\begin{array}{c}
a_{2}+\ldots+a_{l}+1 \\
n
\end{array}\right)+n s_{2}\left(\begin{array}{c}
a_{2}+\ldots+a_{l}+2 \\
n+1
\end{array}\right),
\end{aligned}
$$

from which one gets, by simple computations

$$
\left|O_{3}\right| \leq(l+1)^{n+1} \frac{s_{2}^{n+2}}{s_{3}^{n+1}} .
$$

Now we turn to the calculation of $-\sum_{I_{2}}\left(\begin{array}{l}i-1 \\ n-1\end{array}\right) h(i)$. By [CCD2, loc. cit., (iv)], we have

$$
\sum_{I_{2}}\left(\begin{array}{c}
i-1 \\
n-1
\end{array}\right) h(i)=\sum_{I_{2}}\left(\begin{array}{c}
i-1 \\
n-1
\end{array}\right)\left(1-\pi+i s_{2}\right),
$$

where $\pi:=G^{h}\left(1, r-n ; s_{2}, \ldots, s_{l}\right)$ (when $l=2$, we denote by $G^{h}\left(1, r-n ; s_{2}\right)$ the upper bound for the genus of nondegenerate curves of degree $s_{2}$ in $\mathbf{P}^{r-n}$, found by Castelnuovo $[\mathrm{EH}])$. First notice that, setting

$$
O_{4}:=\left(\begin{array}{c}
a_{2}+\ldots+a_{l}+1 \\
n
\end{array}\right)(1-\pi), \quad O_{5}:=-\left[\left(\begin{array}{c}
a_{1} \\
n
\end{array}\right)-\frac{a_{1}^{n}}{n !}\right](1-\pi),
$$


we have

$$
\sum_{I_{2}}\left(\begin{array}{c}
i-1 \\
n-1
\end{array}\right)(1-\pi)=\frac{a_{1}^{n}}{n !}(1-\pi)-O_{4}-O_{5}
$$

By [CCD2, loc. cit., (1.2)] one has

$$
\pi \leq \frac{s_{2}^{2}}{s_{3}}
$$

Hence we get

$$
\left|O_{4}\right| \leq \frac{l^{n}}{n !} \frac{s_{2}^{n+2}}{s_{3}^{n+1}}
$$

On the other hand, since

$$
\left(\begin{array}{c}
a_{1} \\
n
\end{array}\right)=\frac{1}{n !}\left[a_{1}^{n}-\left(\begin{array}{l}
n \\
2
\end{array}\right) a_{1}^{n-1}\right]+O\left(a_{1}^{n-2}\right), \quad \text { with } \quad\left|O\left(a_{1}^{n-2}\right)\right| \leq(n-2) a_{1}^{n-2},
$$

then

$$
\left|O_{5}\right| \leq\left[\frac{1}{n !}\left(\begin{array}{l}
n \\
2
\end{array}\right) a_{1}^{n-1}+(n-2) a_{1}^{n-2}\right]|1-\pi| \leq(n-1) a_{1}^{n-1}|1-\pi|,
$$

and so, by (A.6), we obtain

$$
\left|O_{5}\right| \leq(n-1) \frac{s_{1}^{n-1}}{s_{2}^{n-3} s_{3}} .
$$

Moreover we have,

$$
\frac{a_{1}^{n}}{n !}(1-\pi)=\frac{1}{n !} \frac{s_{1}^{n}}{s_{2}^{n}}(1-\pi)-O_{6},
$$

where

$$
O_{6}:=-\frac{1-\pi}{n !}\left[\sum_{i=1}^{n}\left(\begin{array}{c}
n \\
i
\end{array}\right)\left(\frac{s_{1}}{s_{2}}\right)^{n-i}\left(\frac{-b_{1}-1}{s_{2}}\right)^{i}\right] \quad \text { and } \quad\left|O_{6}\right| \leq 2 \frac{s_{1}^{n-1}}{s_{2}^{n-3} s_{3}} .
$$

Hence

$$
-\sum_{I_{2}}\left(\begin{array}{c}
i-1 \\
n-1
\end{array}\right)(1-\pi)=-\frac{1}{n !} \frac{s_{1}^{n}}{s_{2}^{n}}(1-\pi)+O_{4}+O_{5}+O_{6} .
$$

Now, in order to estimate $-\sum_{I_{2}} i s_{2}\left(\begin{array}{c}i-1 \\ n-1\end{array}\right)$, we notice that

$$
\begin{gathered}
-\sum_{I_{2}} i s_{2}\left(\begin{array}{c}
i-1 \\
n-1
\end{array}\right) \\
=-\frac{n}{(n+1) !} \frac{s_{1}^{n+1}}{s_{2}{ }^{n}}-\frac{1}{n !} \frac{s_{1}{ }^{n}}{s_{2}{ }^{n}}\left[n\left(s_{2}-b_{1}-1\right)-\frac{n^{2}}{2} s_{2}\right]+O_{7}+O_{8}+O_{9},
\end{gathered}
$$

where

$$
\begin{gathered}
O_{7}:=n s_{2}\left(\begin{array}{c}
a_{2}+\ldots+a_{l}+2 \\
n+1
\end{array}\right) \\
O_{8}:=-n s_{2}\left(\begin{array}{c}
a_{1}+1 \\
n+1
\end{array}\right)+\frac{n s_{2}}{(n+1) !}\left[\left(a_{1}+1\right)^{n+1}-\left(\begin{array}{c}
n+1 \\
2
\end{array}\right)\left(a_{1}+1\right)^{n}\right],
\end{gathered}
$$


and

$$
\begin{aligned}
O_{9}:=-\frac{n s_{2}}{(n+1) !}[ & \sum_{i=2}^{n+1}\left(\begin{array}{c}
n+1 \\
i
\end{array}\right)\left(\frac{s_{1}}{s_{2}}\right)^{n+1-i}\left(\frac{s_{2}-b_{1}-1}{s_{2}}\right)^{i} \\
& \left.-\left(\begin{array}{c}
n+1 \\
2
\end{array}\right) \sum_{i=1}^{n}\left(\begin{array}{c}
n \\
i
\end{array}\right)\left(\frac{s_{1}}{s_{2}}\right)^{n-i}\left(\frac{s_{2}-b_{1}-1}{s_{2}}\right)^{i}\right],
\end{aligned}
$$

with

$$
\left|O_{7}\right| \leq(l+1)^{n+1} \frac{s_{2}^{n+2}}{s_{3}^{n+1}}, \quad\left|O_{8}\right| \leq 2^{n-1} n(n-1) \frac{s_{1}^{n-1}}{s_{2}^{n-2}}, \quad\left|O_{9}\right| \leq 4 n \frac{s_{1}^{n-1}}{s_{2}^{n-2}} .
$$

Consequently, by (A.5), (A.7) and (A.8), we get

$$
\begin{gathered}
-\sum_{I_{2}}\left(\begin{array}{c}
i-1 \\
n-1
\end{array}\right) h(i)=-\frac{1}{n !} \frac{s_{1}^{n}}{s_{2}^{n}}(1-\pi) \\
-\frac{n}{(n+1) !} \frac{s_{1}^{n+1}}{s_{2}^{n}}-\frac{1}{n !} \frac{s_{1}^{n}}{s_{2}^{n}}\left[n\left(s_{2}-b_{1}-1\right)-\frac{n^{2}}{2} s_{2}\right]+\sum_{i=4}^{9} O_{i} .
\end{gathered}
$$

By (A.2), (A.3), (A.4) and (A.9), it follows that

$$
\sum_{I_{1} \cup I_{2}}\left(\begin{array}{c}
i-1 \\
n-1
\end{array}\right)\left(s_{1}-h(i)\right)=\frac{s_{1}{ }^{n+1}}{(n+1) ! s_{2}{ }^{n}}+\frac{s_{1}{ }^{n}}{2 n ! s_{2}{ }^{n-1}}\left[\frac{2 \pi-2}{s_{2}}-n\right]+\sum_{i=1}^{9} O_{i} .
$$

Now we need the explicit expression for $\pi$ given in [CCD2, loc. cit.], i.e. (when $l \geq 3)$

$$
\begin{gathered}
\pi=G^{h}\left(1, r-n ; s_{2}, \ldots, s_{l}\right) \\
=\frac{s_{2}^{2}}{2 s_{3}}+\frac{s_{2}}{2}\left(\frac{s_{3}}{s_{4}}+\ldots+\frac{s_{l}}{r-n+1-l}-l+1-\frac{2}{r-n+1-l}+\eta^{\prime}\right)+\rho^{\prime}+1,
\end{gathered}
$$

where $\eta^{\prime}$ and $\rho^{\prime}$ are rational numbers depending only on $r-n+1-l, s_{3}, \ldots, s_{l}$ and $b_{2}$, such that $\left|\eta^{\prime}\right| \leq \frac{3}{4}, \quad\left|\rho^{\prime}\right| \leq\left[(l-1)^{2}+2(l-1)+9\right] \frac{s_{3}^{3}}{s_{4}^{2}}$. Plugging the previous formula into (A.10), we deduce that

$$
\begin{gathered}
\sum_{I_{1} \cup I_{2}}\left(\begin{array}{c}
i-1 \\
n-1
\end{array}\right)\left(s_{1}-h(i)\right)=\frac{s_{1}{ }^{n+1}}{(n+1) ! s_{2}{ }^{n}} \\
+\frac{s_{1}{ }^{n}}{2 n ! s_{2}{ }^{n-1}}\left[\frac{s_{2}}{s_{3}}+\ldots+\frac{s_{l}}{s_{l+1}}-l-\frac{2}{r-n+1-l}+\eta-(n-1)\right]+\sum_{i=1}^{9} O_{i}
\end{gathered}
$$

where $\eta\left(=\eta^{\prime}+\frac{2 \rho^{\prime}}{s_{2}}\right)$ is exactly the number which appears in the formula for $G^{h}\left(1, r-n+1 ; s_{1}, \ldots, s_{l}\right)$ stated in [CCD2, loc. cit.], and therefore $|\eta| \leq \frac{3}{4}$. By a similar computation, one obtains the same expression for $\sum_{I_{1} \cup I_{2}}\left(\begin{array}{l}i-1 \\ n-1\end{array}\right)\left(s_{1}-h(i)\right)$ in the case of $l=2$.

Hence, by (A.1), to conclude the proof it remains to estimate

$$
O_{10}:=\sum_{I_{3}}\left(\begin{array}{c}
i-1 \\
n-1
\end{array}\right)\left(s_{1}-h(i)\right)
$$


and $\sum_{i=1}^{10} O_{i}$. To do this notice that, by [CCD2, loc. cit.],

$$
\begin{gathered}
O_{10} \leq \sum_{I_{3}}\left(\begin{array}{c}
i-1 \\
n-1
\end{array}\right)\left(s_{1}-h\left(a_{1}\right)\right)=\sum_{I_{3}}\left(\begin{array}{c}
i-1 \\
n-1
\end{array}\right)\left(s_{1}-a_{1} s_{2}-1+\pi\right) \\
=\left(b_{1}+\pi\right) \sum_{i=a_{1}+1}^{a_{1}+\ldots+a_{l}+1}\left(\begin{array}{c}
i-1 \\
n-1
\end{array}\right) .
\end{gathered}
$$

In order to evaluate $\sum_{i=a_{1}+1}^{a_{1}+\ldots+a_{l}+1}\left(\begin{array}{c}i-1 \\ n-1\end{array}\right)$ and to simplify the notation, put $\alpha:=$ $a_{2}+\ldots+a_{l}+1$. One has

$$
\begin{gathered}
\sum_{i=a_{1}+1}^{a_{1}+\ldots+a_{l}+1}\left(\begin{array}{c}
i-1 \\
n-1
\end{array}\right)=\sum_{i=0}^{\alpha-1}\left(\begin{array}{c}
a_{1}+\alpha-i-1 \\
n-1
\end{array}\right) \\
\leq \alpha\left(\begin{array}{c}
a_{1}+\alpha-1 \\
n-1
\end{array}\right) \leq \frac{\alpha}{(n-1) !}\left(a_{1}+\alpha\right)^{n-1} \leq 2^{n-1} \frac{\alpha}{(n-1) !} a_{1}^{n-1} \leq 2 \alpha a_{1}^{n-1} .
\end{gathered}
$$

Hence we get, by (A.6) and (A.11),

because $\alpha \leq l a_{2}$.

$$
\left|O_{10}\right| \leq\left(\frac{s_{2}^{2}}{s_{3}}+s_{2}\right)\left(2 \alpha a_{1}^{n-1}\right) \leq 4 l \frac{s_{1}^{n-1}}{s_{2}^{n-4} s_{3}^{2}}
$$

Finally, put

$$
O:=\sum_{i=1}^{10} O_{i}
$$

and note that, by using all previous bounds for the $O_{i}$ 's, one has

$$
|O| \leq s_{1}^{n-1} \frac{s_{2}^{4-n}}{s_{3}^{2}}\left[4(l+1)^{n+1}+2^{n-1} n(n+8)\right] .
$$

Proof of Proposition B. First we analyse the inequality (ii) for $i=2$. To do this notice that by Proposition A we get

$$
\begin{gathered}
G^{h}\left(n, r ; s_{1}, \ldots, s_{l}\right)-G^{h}\left(n, r ; s_{1}, s_{2}+1\right) \\
\geq \frac{s_{1}{ }^{n}}{2 n ! s_{2}{ }^{n-1}}\left\{\frac{2 s_{1}}{(n+1) s_{2}\left(s_{2}+1\right)}-l-n-\frac{7}{2}+\frac{\left(O-O^{\prime}\right) 2 n ! s_{2}{ }^{n-1}}{s_{1}{ }^{n}}\right\},
\end{gathered}
$$

where $O^{\prime}$ has, with respect to $G^{h}\left(n, r ; s_{1}, s_{2}+1\right)$, the same meaning as $O$ has with respect to $G^{h}\left(n, r ; s_{1}, \ldots, s_{l}\right)$. Now, by taking into account our numerical assumption (1.4.4), again by Proposition A we have

$$
\left|\frac{\left(O-O^{\prime}\right) 2 n ! s_{2}{ }^{n-1}}{s_{1}{ }^{n}}\right| \leq \frac{1}{2},
$$

and so, continuing our calculations, it follows that

$$
\begin{aligned}
& G^{h}\left(n, r ; s_{1}, \ldots, s_{l}\right)-G^{h}\left(n, r ; s_{1}, s_{2}+1\right) \\
& \geq \frac{s_{1}^{n}}{2 n ! s_{2}^{n-1}}\left[\frac{2 s_{1}}{(n+1) s_{2}\left(s_{2}+1\right)}-(l+n+4)\right]
\end{aligned}
$$


which is $\delta_{2}$. In a similar way one analyses the case $3 \leq i \leq l$. Finally we notice that property (i) is an immediate consequence of our numerical assumption $s_{1}>>$ $\ldots>>s_{l}$.

\section{REFERENCES}

[ACGH] E.Arbarello, M.Cornalba, P.A.Griffiths and J.Harris, Geometry of algebraic curves, Volume I, Springer-Verlag, Berlin, 1985. MR 86h:14019

[BF] R.Braun and G.Fløystad, A bound for the degree of smooth surfaces in $\mathbf{P}^{4}$ not of general type, Compositio Mathematica 93 (1994), 211-229. MR 95g:14037

[Ca] G.Castelnuovo, Ricerche di geometria sulle curve algebriche, Zanichelli, Bologna, 1937.

[CC] L.Chiantini and C.Ciliberto, A few remarks on the lifting problem, Journées de Géométrie Algébrique d'Orsay (1992), Astérisque, no. 218, Soc. Math. France, Paris, 1993, pp. 95109. MR 95c: 14072

[CCD1] L.Chiantini, C.Ciliberto and V.Di Gennaro, The genus of projective curves, Duke Math. J. 70 (1993), 229-245. MR 94b:14027

[CCD2] L.Chiantini, C.Ciliberto and V.Di Gennaro, On the genus of projective curves verifying certain flag conditions, preprint, 1995.

[CCD3] L.Chiantini, C.Ciliberto and V.Di Gennaro, The genus of curves in $\mathbf{P}^{4}$ verifying certain flag conditions, Manuscripta Math. 88 (1995), 119-134. MR 96h:14044

[Ci1] C.Ciliberto, Hilbert functions on finite sets of points and the genus of a curve in a projective space, Space Curves: Proceedings, Rocca di Papa, 1985, Lecture Notes in Math., vol. 1266, Springer-Verlag, Berlin, 1987, pp. 24-73. MR 89c:14039

[Ci2] C.Ciliberto, On a property of Castelnuovo varieties, Transactions of the American Mathematical Society 303/1 (1987), 201-210. MR 88e:14055

[Ci3] C.Ciliberto, Alcune applicazioni di un classico procedimento di Castelnuovo, Seminari di Geometria 1982-1983, Università di Bologna, Bologna, 1984, pp. 17-43. MR 86j:14021

[D] V.Di Gennaro, Generalized Castelnuovo varieties, manuscripta math. 81 (1993), 311328. MR 95f: 14074

[EH] D.Eisenbud and J.Harris, Curves in projective space, Sém. Math. Sup., vol. 85, Les Presses de l'Université de Montréal, Montréal, vol. 85, 1982. MR 84g:14024

[E] P.Ellia, Sur les lacunes d'Halphen, Algebraic Curves and Projective Geometry: Proceedings, Trento, 1988, Lecture Notes in Math., vol. 1389, Springer-Verlag, Berlin, 1989, pp. 43-65. MR 90j:14031.

[EP] G.Ellingsrud and C.Peskine, Sur les surfaces lisses de $\mathbf{P}_{4}$, Invent. Math. 95 (1989), 1-11. MR 89j: 14023

[GH] P.A.Griffiths and J.Harris, Principles of algebraic geometry, Wiley, New York, 1978. MR 80b: 14001

[GLP] L.Gruson, R.Lazarsfeld and C.Peskine, On a Theorem of Castelnuovo, and the Equations Defining Space Curves, Invent. Math. 72 (1983), 491-506.

[GP] L.Gruson and C.Peskine, Genre des courbes dans l'espace projectif, Algebraic Geometry: Proceedings, Norway, 1977, Lecture Notes in Math., vol. 687, Springer Verlag, New York, 1978, pp. 31-59. MR 81e:14019

[Ha] G.Halphen, Mémoire sur la classification des courbes gauches algébriques, Oeuvres complètes t.III.

[Hr1] J.Harris, The genus of space curves, Math. Ann. 249 (1980), 191-204. MR 81i:14022

[Hr2] J.Harris, A bound on the geometric genus of projective varieties, Ann. Scuola Norm. Sup. Pisa Cl. Sci. (4) 8 (1981), 35-68. MR 82h:14010

[Ht] R.Hartshorne, Algebraic geometry, Springer-Verlag, Berlin, 1977. MR 57:3116

[J] F.Jongmans, Contribution à la théorie des variétés algébriques, Thèse d'Agrégation de l'Einseignement supérieur, Bruxelles, M.Hayez, Imprimeur de l'Académie Royale de Belgique 1947; also published as Mém. Soc. Roy. Sci. Liège (4) 7 (1947), 367-468. MR 9:611c

[K] E.Kunz, Holomorphe Differenzialformen auf algebraischen Varietäten mit Singularitäten. I, Manuscripta Math. 15 (1975), 91-108. MR 52:5674

[M] D.Mumford, Lectures on Curves on an Algebraic Surface, Ann. of Math. Stud., Princeton Univ. Press, Princeton, N.J., 1966. MR 35:187 
[N] U.Nagel, On bounds for cohomological Hilbert functions, notes from author's thesis "Über Gradschranken für Syzygien und kohomologische Hilbertfunktionnen", Paderborn, 1990, J. Algebra 150 (1992), 231-244. MR 93i:13011

[NV] U.Nagel and W.Vogel, Bounds for Castelnuovo's regularity and Hilbert functions, Topics in Algebra, Banach Center Publ., vol. 26; part 2, PWN, Warsaw (1990), pp. 163-183. MR 93k:13024

[No] M.Noether, Zur Grundlegung der Theorie der algebraischen Raumcurven, Königlichen Akad. der Wissenschaften, 1883.

[PS] C.Peskine and L.Szpiro, Liaison des variétés algébriques. I, Inventiones Math. 26 (1974), 271-302. MR 51:526

[Re] R.Re, Sulle sezioni iperpiane di una varietà proiettiva, Le Matematiche (Catania) 42 (1987), 211-218 (1989). MR 90j:14069

[R] M.Reid, Canonical 3-folds, Journées de Géométrie Algébrique d'Angers 1979, Sijthoff \& Noordhoff, Alphen aan den Rijn, 1980, pp. 273-310. MR 82i:14025.

[Se] E.Sernesi, Topics on families of projective schemes, Queen's Papers Pure Appl. Math., vol. 73, Queen's Univ., Kingston, Ont., 1986. MR 88b:14006

[Sv] F.Severi, Intorno ai punti doppi improprii di una superficie generale dello spazio a 4 dimensioni e a suoi punti tripli apparenti, Rendiconti di Palermo 15 (1901).

[St] R.Strano, On generalized Laudal's lemma, Complex Projective Geometry, (Trieste and Bergen, 1989; G. E. Ellingsrud et al., eds.), London Math. Soc. Lecture Note Ser., vol. 179, Cambridge Unov. Press, Cambridge, 1992, pp. 284-293. MR 94a:14032

Dipartimento di Matematica, Università di Roma "Tor Vergata”, 00133 Roma, Italy

E-mail address: Digennaro@mat.utovrm.it 